Supporting Information for:

\title{
Homoleptic Tris-Diphosphine Re(I) and Re(II) Complexes and Re(II) Photophysics and Photochemistry
}

Jeramie J. Adams, Rose Donohoue, Navamoney Arulsamy, B. Pat Sullivan, Dean M. Roddick, ${ }^{*}$ Amelia Neuberger, ${ }^{\dagger}$ and Russell Schmehl, ${ }^{+*}{ }^{*}$

Department of Chemistry, Box 3838

University of Wyoming, Laramie, Wyoming 82071, U. S. A.

and

Department of Chemistry, Tulane University, New Orleans, Louisiana $70118^{\dagger}$

\section{Contents:}

A. Crystallographic Data Collection and Refinement

Table S1. Crystallographic Data for $\left[\mathrm{Re}(\mathrm{dmpe})_{3}\right]\left(\mathrm{B}\left(\mathrm{C}_{6} \mathrm{~F}_{5}\right)_{4}\right) \cdot \mathrm{CH}_{2} \mathrm{Cl}_{2}(\mathbf{1})$ and [Re(depe) $\left.)_{3}\right] \mathrm{B}(\mathrm{C} 6 \mathrm{~F} 5)_{4}$ (2).

Table S2. Crystallographic Data for $\left[\mathrm{Re}(\mathrm{dmpm})_{3}\right]\left(\mathrm{B}\left(\mathrm{C}_{6} \mathrm{~F}_{5}\right)_{4}\right) \cdot(3)$ and fac$\left[\mathrm{Re}\left(\mathrm{Me}_{2} \mathrm{PCH}_{2} \mathrm{PPh}_{2}\right)_{3}\right]\left(\mathrm{B}\left(\mathrm{C}_{6} \mathrm{~F}_{5}\right)_{4}\right) \cdot(4)$.

Table S3. Crystallographic Data for $\left[\mathrm{Re}(\mathrm{dmpp})_{3}\right]\left(\mathrm{B}\left(\mathrm{C}_{6} \mathrm{~F}_{5}\right)_{4}\right) \cdot \mathrm{CH}_{2} \mathrm{Cl}_{2}(5)$ and $\left[\operatorname{Re}(\mathrm{dmpb})_{3}\right] \mathrm{B}(\mathrm{C} 6 \mathrm{~F} 5) 4$ (7).

Table S4. Crystallographic Data for $\left[\mathrm{Re}(\mathrm{dmpe})_{3}\right]\left(\mathrm{B}\left(\mathrm{C}_{6} \mathrm{~F}_{5}\right)_{4}\right)_{2} \cdot 3 \mathrm{MeCN}(\mathbf{8})$ and $\left[\mathrm{Re}(\mathrm{depe})_{3}\right]$ $\left(\mathrm{B}\left(\mathrm{C}_{6} \mathrm{~F}_{5}\right)_{4}\right)_{2} \cdot 2 \mathrm{MeOH}(\mathbf{9})$.

Table S5. Crystallographic Data for $\left[\mathrm{Re}(\mathrm{dmpm})_{3}\right]\left(\mathrm{B}\left(\mathrm{C}_{6} \mathrm{~F}_{5}\right)_{4}\right)_{2} \cdot \mathrm{CH}_{2} \mathrm{Cl}_{2}(\mathbf{1 0})$ and $\left[\mathrm{Re}\left(\mathrm{Me}_{2} \mathrm{PCH}_{2} \mathrm{PPh}_{2}\right)_{3}\right]_{2}\left(\mathrm{PF}_{6}\right)_{3}(\mathbf{1 1})$.

Table S6. Crystallographic Data for $\left[\mathrm{Re}(\mathrm{dmpb})_{3}\right]\left(\mathrm{SbF}_{6}\right)_{2} \cdot \mathrm{PhCl}(\mathbf{1 4})$.

Table S7. Crystallographic Data for $\left[\mathrm{Re}(\mathrm{dmpm})_{3}(\mathrm{MeCN})\right]\left(\mathrm{SbF}_{6}\right)_{3}(\mathbf{1 5})$

Table S8. Selected Bond Distances $(\AA)$ and Angles $\left(^{\circ}\right)$ for $\left[\operatorname{Re}(\mathrm{dmpe})_{3}\right] \mathrm{B}\left(\mathrm{C}_{6} \mathrm{~F}_{5}\right)_{4} \cdot \mathrm{CH}_{2} \mathrm{Cl}_{2}(\mathbf{1})$.

Table S9. Selected Bond Distances $(\AA)$ and Angles $\left({ }^{\circ}\right)$ for $\left[\operatorname{Re}(\text { depe })_{3}\right] \mathrm{B}\left(\mathrm{C}_{6} \mathrm{~F}_{5}\right)_{4}(\mathbf{2})$.

Table S10. Selected Bond Distances $(\AA)$ and Angles $\left({ }^{\circ}\right)$ for $\left[\operatorname{Re}(\mathrm{dmpm})_{3}\right] \mathrm{B}\left(\mathrm{C}_{6} \mathrm{~F}_{5}\right)_{4}(3)$.

Table S11. Selected Bond Distances $(\AA \AA)$ and Angles $\left({ }^{\circ}\right)$ for $f a c-\left[\operatorname{Re}\left(\mathrm{Me}_{2} \mathrm{PCH}_{2} \mathrm{PPh}_{2}\right)_{3}\right]$ $\mathrm{B}\left(\mathrm{C}_{6} \mathrm{~F}_{5}\right)_{4}(\mathbf{4})$.

Table S12. Selected Bond Distances $(\AA ̊)$ and Angles $\left({ }^{\circ}\right)$ for $\left[\operatorname{Re}(d m p p)_{3}\right] B\left(C_{6} F_{5}\right)_{4}(\mathbf{5})$.

Table S13. Selected Bond Distances $(\AA)$ and Angles $\left({ }^{\circ}\right)$ for $\left[\operatorname{Re}(d m p p)_{3}\right] B\left(C_{6} F_{5}\right)_{4}(7)$. 
Table S14. Selected Bond Distances $(\AA \AA)$ and Angles $\left({ }^{\circ}\right)$ for $\left[\operatorname{Re}(\mathrm{dmpe})_{3}\right]\left(\mathrm{B}\left(\mathrm{C}_{6} \mathrm{~F}_{5}\right)_{4}\right)_{2} \cdot 3 \mathrm{MeCN}$ (8).

Table S15. Selected Bond Distances $(\AA)$ and Angles $\left({ }^{\circ}\right)$ for $\left[\operatorname{Re}(\text { depe })_{3}\right]\left(B\left(\mathrm{C}_{6} \mathrm{~F}_{5}\right)_{4}\right)_{2} \cdot 2 \mathrm{MeOH}$ (9).

Table S16. Selected Bond Distances $(\AA)$ and Angles $\left({ }^{\circ}\right)$ for $\left[\operatorname{Re}(\mathrm{dmpm})_{3}\right]\left(\mathrm{B}\left(\mathrm{C}_{6} \mathrm{~F}_{5}\right)_{4}\right)_{2} \cdot \mathrm{CH}_{2} \mathrm{Cl}_{2}$ (10).

Table S17. Selected Bond Distances $(\AA)$ and Angles $\left({ }^{\circ}\right)$ for $\left[\mathrm{Re}\left(\mathrm{Me}_{2} \mathrm{PCH}_{2} \mathrm{PPh}_{2}\right)_{3}\right]_{2}\left(\mathrm{PF}_{6}\right)_{3}(\mathbf{1 1})$.

Table S18. Selected Bond Distances $(\AA)$ and Angles $\left({ }^{\circ}\right)$ for $\left[\mathrm{Re}(\mathrm{dmpb})_{3}\right]\left(\mathrm{SbF}_{6}\right)_{2} \cdot \mathrm{PhCl}(\mathbf{1 4})$.

Table S19. Cartesian coordinates (in $\AA$ ) of optimized geometry for (dmpe) ${ }_{3} \mathrm{Re}^{+}$(DFT M06-L functional, cc-pVDz atom basis sets)

Table S20. Cartesian coordinates (in $\AA$ ) of optimized geometry for (dmpe) ${ }_{3} \operatorname{Re}^{2+}$ (DFT M06-L functional, cc-pVDz atom basis sets)

B. Absorption Spectra of Complexes

Figure S1. UV-Vis spectra of $(\mathrm{PP})_{3} \mathrm{Re}^{2+}$ in $\mathrm{CH}_{3} \mathrm{CN}(\mathrm{PP}=\mathrm{dmpm}$, dmpe, dmpp ).

Figure S2. UV-Vis spectra of (PP) ${ }_{3} \mathrm{Re}^{2+}$ in $\mathrm{CH}_{3} \mathrm{CN}\left(\mathrm{PP}=\mathrm{dmpm}, \mathrm{Me}_{2} \mathrm{PCH}_{2} \mathrm{PPh}_{2}\right.$, dppm).

Figure S3. UV-Vis spectra of $(\mathrm{PP}){ }_{3} \mathrm{Re}^{2+}$ in $\mathrm{CH}_{3} \mathrm{CN}(\mathrm{PP}=\mathrm{dmpe}$, depe, dmpb)

C. Room temperature emission spectra of complexes

Figure S4. Uncorrected emission spectra for a deoxygenated $0.23 \mathrm{mM}$ solution of $\left[(\mathrm{PP})_{3} \mathrm{Re}\right]^{2+}$, for phosphines containing methyl or ethyl phosphine substituents, in $\mathrm{CH}_{3} \mathrm{CN}$ at room temperature.

D. Cyclic Voltammetric Data for Several Complexes

Figure S5. Cyclic Voltammetry for $(\mathrm{PP})_{3} \mathrm{Re}^{+}$complexes $\left(\mathrm{PP}=\mathrm{dmpm}, \mathrm{Me}_{2} \mathrm{PCH}_{2} \mathrm{PPh}_{2}\right.$, dppm ) in $1 \mathrm{mM} \mathrm{CH}_{3} \mathrm{CN}$.

Figure S6. Cyclic Voltammetry for $(\mathrm{PP})_{3} \mathrm{Re}^{+}$complexes ( $\mathrm{PP}=\mathrm{dmpm}, \mathrm{dmpp}$, dmpe) in $1 \mathrm{mM} \mathrm{CH} \mathrm{CH}_{3} \mathrm{CN}$

E. $\quad 77 \mathrm{~K}$ Emission Spectra of the complexes in Propionitrile/Butyronitrile matrices. 
Figure S7. $77 \mathrm{~K}$ luminescence spectrum of $\left[\operatorname{Re}(\mathrm{dmpp})_{3}\right]^{2+}$ in a mixed propionitrile/ butyronitrile matrix.

Figure S8. $77 \mathrm{~K}$ luminescence spectrum of $\left[\operatorname{Re}(\mathrm{dmpe})_{3}\right]^{2+}$ in a mixed propionitrile/ butyronitrile matrix.

Figure S9. $77 \mathrm{~K}$ luminescence spectrum of $\left[\operatorname{Re}(\mathrm{dmpm})_{3}\right]^{2+}$ in a mixed propionitrile/ butyronitrile matrix.

Figure S10. 77K luminescence spectrum of $\left[\mathrm{Re}\left(\mathrm{Me}_{2} \mathrm{PCH}_{2} \mathrm{PPh}_{2}\right)_{3}\right]^{2+}$ in a mixed propionitrile/ butyronitrile matrix.

Figure S11. 77K luminescence spectrum of $\left[\operatorname{Re}(\mathrm{dmpb})_{3}\right]^{2+}$ in a mixed propionitrile/ butyronitrile matrix.

F. Figure S12. Infrared spectra of $\left[\operatorname{Re}(\mathrm{dmpe})_{3}\right]^{+},\left[\operatorname{Re}(\mathrm{dmpe})_{3}\right]^{2+}$ and $\left[\operatorname{Re}(\mathrm{dmpp})_{3}\right]^{2+}$.

G. Figure S13. Plot of the absorption maxima and luminescence maxima of the complexes as a function of $\mathrm{E}^{0}\left(\mathrm{Re}^{2+/+}\right)$.

H. Table S19. Luminescence quenching rate constants for $\left[\operatorname{Re}(\text { depe })_{3}\right]^{2+}$ by a series of amines, alkoxybenzenes and simple aromatics. 


\section{Crystallographic Data Collection and Refinement}

$\mathrm{X}$-ray quality crystals were grown as described in the experimental section. Crystals were mounted in hydrocarbon oil on a MiTeGen micromount or Hampton Research cryoloop or glass fiber. The data were collected at $150 \mathrm{~K}$ on a Bruker SMART APEX II CCD area detector system equipped with a graphite monochromator and a Mo Ka fine-focus sealed tube operated at $1.5 \mathrm{~kW}$ power $(50 \mathrm{kV}, 30 \mathrm{~mA})$. The detector was either placed at $5.12 \mathrm{~cm}$ from the crystals. In each case, a series of narrow frames of data were collected with a scan width of $0.5^{\circ}$ in $\omega$ or $\phi$ and an exposure time of $10 \mathrm{~s}$ per frame. The frames were integrated with the Bruker SAINT software program using a narrow-frame integration algorithm. ${ }^{1}$ The data were corrected for absorption effects by either the semiempirical multi-scan (for 1, 2, 3, 5, 7, 8, 9, 11, and 14) or the numerical face-indexing methods (for 4, 10) using SADABS. ${ }^{1}$ Crystallographic data collection parameters and refinement data are collected below in Tables S1 - S5. All of the structures were solved by the Direct methods using the Bruker SHELXTL (V. 6.14) Software package. ${ }^{1}$

Compound $\mathbf{1}$ crystallizes in space group $P \overline{1}$. All non-hydrogen atoms were located in successive Fourier maps and refined anisotropically. The asymmetric unit consists of a $\left[\operatorname{Re}(\mathrm{dmpe})_{3}\right]^{+}$cation, one tetrakis(perfluorophenyl)borate anions and a solvated dichloromethane molecule. Three of the dmpe carbon atoms exhibit minor positional disorder. However, no other positions for the atoms could be located in the Fourier maps and the current refinement is satisfactory. The hydrogen atoms were placed in calculated positions and refined isotropically adopting a riding model.

Compound 2 crystallizes in space group $P \overline{1}$. All non-hydrogen atoms were located in successive Fourier maps and refined anisotropically. The hydrogen atoms were placed in calculated positions and refined isotropically adopting a riding model. The asymmetric unit consists of a $\left[\operatorname{Re}(\mathrm{depe})_{3}\right]^{+}$cation and one tetrakis(perfluorophenyl)borate anion. The ions are well ordered and well separated from each other.

Compound 3 crystallizes in space group $P \overline{1}$. All non-hydrogen atoms were located in successive Fourier maps and refined anisotropically. The asymmetric unit consists of a $\left[\operatorname{Re}(\mathrm{dmpm})_{3}\right]^{+}$complex cation, and a tetrakis(pentafluorophenyl)borate anion. Both ions are located on general positions and well separated. Whereas the anion is well ordered, the cation exhibits significant disorder: two of the dmpm ligands are disordered. The disorder was treated by assigning the two dmpm ligands two sets of positions. This model led to a satisfactory refinement of the overall structure. All hydrogen atoms were placed in calculated positions and refined adapting a riding model with fixed thermal parameters.

Compound 4 crystallizes in space group $P 2_{1} / n$. All non-hydrogen atoms were located in successive Fourier maps and refined anisotropically. The asymmetric unit consists of a $\left[\mathrm{Re}\left(\mathrm{Me}_{2} \mathrm{PCH}_{2} \mathrm{PPh}_{2}\right)_{3}\right]^{+}$cation and a tetrakis(pentafluorophenyl)borate anion. The ions are well ordered and well separated from each other. All hydrogen atoms were placed in calculated positions and refined isotropically adopting a riding model.

Compound $\mathbf{5}$ crystallizes in space group $P \overline{1}$. The asymmetric unit consists of three each of the $\left[\operatorname{Re}(\mathrm{dmpp})_{3}\right]^{+}$cations and tetrkis(pentafluorophenyl)borate anions. Whereas all 
of the anions and one of the cations are well ordered the rest of the cations exhibit significant disorder. No satisfactory model could be applied to treat the disorder, and so the overall refinement is not accurate. Yet we believe the metric parameters associated with the well ordered $\left[\operatorname{Re}(\mathrm{dmpp})_{3}\right]^{+}$are reliable. The hydrogen atoms were placed in calculated positions and refined isotropically adopting a riding model with fixed positional parameters. No hydrogen atoms were added to the disordered methyl and methylene groups of the two disordered cations. The solvent hydrogen atoms were neither located nor placed in calculated positions. The three $\left[\operatorname{Re}(\mathrm{dmpp})_{3}\right]^{+}$cations are chemically identical and are crystallographically unique due to varying degrees of disorder. The roughly spherical shape of the cation and its relatively smaller size in comparison to the borate anions probably account for the disorder. The ions are also well separated allowing the spherical cations to freely rotate in the crystal lattice.

Compound 7 crystallizes in the chiral monoclinic space group $C c$. The asymmetric unit consists of two $\left[\operatorname{Re}(\mathrm{dmpb})_{3}\right]^{+}$cations and two tetrakis(pentafluoro)borate anions. The ions are well ordered and located on general positions. All of the hydrogen atoms were placed in calculated positions and refined isotropically adopting a riding model with fixed positional parameters. The absolute structure could not be determined accurately as the Flack parameter is refined to $0.634(3)$, which deviates from the expected value of zero significantly. ${ }^{2}$

Compound 8 crystallizes in space group $P \overline{1}$. All non-hydrogen atoms were located in successive Fourier maps and refined anisotropically. The hydrogen atoms were placed in calculated positions and refined isotropically adopting a riding model. The asymmetric unit consists of a $\left[\operatorname{Re}\left(\mathrm{dmpe}_{3}\right]^{2+}\right.$ cation, two tetrakis(perfluorophenyl)borate anions and three solvated acetonitrile molecules. The cation exhibits minor disorder of some of the dmpe carbon atoms, but the overall refinement of the structure is satisfactory. The anions and the solvates are well ordered.

Compound 9 crystallizes in space group $P 2_{1} / c$. All non-hydrogen atoms were located in successive Fourier maps and refined anisotropically. The hydrogen atoms were placed in calculated positions and refined isotropically adopting a riding model. The asymmetric unit consists of a $\left[\operatorname{Re}(\text { depe })_{3}\right]^{2+}$ cation, two tetrakis(perfluorophenyl)borate anions and two solvent methanol solvents. The cation and the anions are well ordered and well separated, but the solvent molecules are disorder. The disorder was satisfactorily modeled with two sets of positions for the methanol molecules.

Compound 10 crystallizes in space group $P 21 / c$. All non-hydrogen atoms were located in successive Fourier maps and refined anisotropically. The asymmetric unit consists of a $\left[\operatorname{Re}(\mathrm{dmpm})_{3}\right]^{2+}$ cation, two tetrakis(pentafluorophenyl)borate anions and a dichloromethane molecule. The cations and the anion are well ordered, whereas the solvent molecule is disordered over two sites. All hydrogen atoms except those of the dichloromethane molecule were placed in calculated positions and refined isotropically adopting a riding model. The latter hydrogen atoms were neither located nor placed in calculated positions.

Compound $\mathbf{1 1}$ crystallizes in space group $\mathrm{Pa} \overline{3}$. All non-hydrogen atoms were located in successive Fourier maps and refined anisotropically. The asymmetric unit 
consists of one third of the $\left[\mathrm{Re}\left(\mathrm{Me}_{2} \mathrm{PCH}_{2} \mathrm{PPh}_{2}\right)_{3}\right]^{1+/ 2+}$ cation and a $\mathrm{PF}_{6}$ - located on a threefold symmetry axis, and another $\mathrm{PF}_{6}{ }^{-}$anion located on a six-fold symmetry axis. Hence the stoichiometry of the cation and anion is 2:3. Based on the stoichiometry, we propose that the crystals contain both the $\left[\mathrm{Re}\left(\mathrm{Me}_{2} \mathrm{PCH}_{2} \mathrm{PPh}_{2}\right)_{3}\right]^{1+}$ and $\left[\mathrm{Re}\left(\mathrm{Me}_{2} \mathrm{PCH}_{2} \mathrm{PPh}_{2}\right)_{3}\right]^{2+}$ cations, and that the two possess comparable structural features. The cation and the anion located on the six-fold axis are well ordered. The other anion exhibits some disorder: the four equatorial $\mathrm{F}$ atoms occupy 6 positions with a partial occupancy. The phenyl hydrogen atoms were located in the Fourier maps and refined isotropically. The rest of the hydrogen atoms were placed in calculated positions and refined isotropically adopting a riding model.

Compound 14 crystallizes in the chiral non-centrosymmetric space group $P 2{ }_{1}$. The asymmetric unit consists of two $\left[\operatorname{Re}(\mathrm{dmpb})_{3}\right]^{2+}$ cations, four hexafluoroantimony anions and one chlorobenzene solvent molecule. All of them are well ordered and located on general positions. All hydrogen atoms were placed in calculated positions and refined isotropically adopting a riding model with fixed positional parameters. The absolute structure is determined accurately by refining the Flack parameter to a value close to zero, $-0.003(3) .^{2}$

Compound 15 crystallizes in the monoclinic space group C2/c. All non-hydrogen atoms were located in successive Fourier maps and refined anisotropically. The asymmetric unit consists of a $\left[\mathrm{Re}(\mathrm{dmpm})_{3}(\mathrm{MeCN})\right]^{3+}$ complex cation and two $\mathrm{SbF}_{6}$ anions on general positions and two $\mathrm{SbF}_{6}$ anions located on a two-fold symmetry axis. The complex cation and the two anions located on general positions along with one of the $\mathrm{SbF}_{6}{ }^{-}$ anions on a special position were well ordered. The other anion is highly disordered, and the disorder is treated by assigning 12 sites of partial occupancy for the 6 fluorine atoms. The hydrogen atoms were placed in calculated positions and refined isotropically adopting a riding model with fixed positional parameters.

\section{References}

1. APEX2 Software Suite, Bruker AXS: Madison, WI, 2008.

2. Flack, H. D. Acta Crystallogr. Sect. A. 1983, A39, 876-881. 
Table S1. Crystallographic Data for $\left[\mathrm{Re}(\mathrm{dmpe})_{3}\right] \mathrm{B}\left(\mathrm{C}_{6} \mathrm{~F}_{5}\right)_{4} \cdot \mathrm{CH}_{2} \mathrm{Cl}_{2}(\mathbf{1})$ and $\left[\mathrm{Re}(\mathrm{depe})_{3}\right]$ $\mathrm{B}\left(\mathrm{C}_{6} \mathrm{~F}_{5}\right)_{4}(2)$.

12

CCDC

Empirical formula

$\mathrm{C}_{43} \mathrm{H}_{50} \mathrm{BCl}_{2} \mathrm{~F}_{20} \mathrm{P}_{6} \mathrm{Re}$

$\mathrm{C}_{54} \mathrm{H}_{72} \mathrm{BF}_{20} \mathrm{P}_{6} \mathrm{Re}$

Color

Colorless

Colorless

Formula weight

1400.56

1483.95

Crystal dimensions

$0.483 \times 0.330 \times 0.286$

$0.30 \times 0.15 \times 0.14$

Crystal system, space group

Triclinic, $P \overline{1}$

Triclinic, $P \overline{1}$

$a(\AA)$

10.1455 (1)

$10.5112(4)$

$b(\AA)$

16.3292(2)

$15.3343(5)$

$c(\AA)$

17.6183(2)

18.9462(7)

$\alpha\left(^{\circ}\right)$

63.25

84.663(2)

$\beta\left(^{\circ}\right)$

$79.856(1)$

$80.836(2)$

$\gamma\left({ }^{\circ}\right)$

$89.389(1)$

78.621(2)

$V\left(\AA^{3}\right)$

2557.75(5)

2949.6(2)

Z

2

2

$\rho_{\text {calcd }}\left(\mathrm{g} \mathrm{cm}^{-1}\right)$

1.819

1.671

$\mu\left(\mathrm{mm}^{-1}\right)$

2.774

2.323

$2 \theta \operatorname{limit}\left({ }^{\circ}\right)$

2.21 to 32.58

3.37 to 28.28

Index ranges

$-15 \leq h \leq 12$

$-14 \leq h \leq 14$

$-24 \leq k \leq 24$

$-20 \leq k \leq 19$

$-26 \leq l \leq 26$

$-25 \leq l \leq 25$

Independent reflections $\left(R_{\text {int }}\right)$

$18337(0.0571)$

$14273(0.0285)$

Data/restraints/parameters

$18337 / 0 / 658$

14273 / 0 / 739

Goodness-of-fit

1.058

1.030

$R 1[I>2 \sigma(I)]^{a}$

0.0348

0.0253

$w R 2[I>2 \sigma(I)]^{\mathrm{b}}$

0.0950

0.0554

Largest peak, hole $\left(\mathrm{e}^{-3}\right)$

4.208, -2.080

$1.052,-0.398$ 
Table S2. Crystallographic Data for $\left[\operatorname{Re}(\mathrm{dmpm})_{3}\right] \mathrm{B}\left(\mathrm{C}_{6} \mathrm{~F}_{5}\right)_{4}(3)$ and fac- $\left[\mathrm{Re}\left(\mathrm{Me}_{2} \mathrm{PCH}_{2} \mathrm{PPh}_{2}\right)_{3}\right]$ $\mathrm{B}\left(\mathrm{C}_{6} \mathrm{~F}_{5}\right)_{4}(4)$.

3

$\overline{\text { CCDC }}$

Empirical formula

Color

Formula weight

Crystal dimensions

Crystal system, Space group

$a(\AA)$

$b(\AA)$

$c(\AA)$

$\alpha\left(^{\circ}\right)$

$\beta\left(^{\circ}\right)$

$\gamma\left({ }^{\circ}\right)$

$V\left(\AA^{3}\right)$

$Z$

$\rho_{\text {calcd }}\left(\mathrm{g} \mathrm{cm}^{-1}\right)$

$\mu\left(\mathrm{mm}^{-1}\right)$

$2 \theta \operatorname{limit}\left({ }^{\circ}\right)$

Index ranges

Independent reflections $\left(R_{\text {int }}\right)$

Data/restraints/parameters

Goodness-of-fit

$R 1[I>2 \sigma(I)]^{a}$

$w R 2[I>2 \sigma(I)]^{\mathrm{b}}$

Largest peak, hole $\left(\mathrm{e}^{-3}\right)$
$\mathrm{C}_{39} \mathrm{H}_{42} \mathrm{BF}_{20} \mathrm{P}_{6} \mathrm{Ru}$

Colorless

1273.56

$0.44 \times 0.36 \times 0.34$

Triclinic, $P \overline{1}$

13.4704(1)

14.8232(3)

14.9949(2)

103.416(1)

116.598(1)

105.723(1)

2349.88(6)

2

1.800

2.900

$1.76-33.73$

$-21 \leq h \leq 21$

$-23 \leq k \leq 23$

$-22 \leq l \leq 23$

18584(0.0201)

18584 / 0 / 726

1.053

0.0325

0.0793

$2.442,-1.440$
4

$\mathrm{C}_{69} \mathrm{H}_{54} \mathrm{BF}_{20} \mathrm{P}_{6} \mathrm{Re}$

Yellow

1645.95

$0.27 \times 0.21 \times 0.15$

Monoclinic, $P 2_{1} / n$

11.0920(3)

33.3161(1)

18.1664(5)

90

106.266(2)

90

6444.5(3

4

1.696

2.137

$2.13-33.14$

$-14 \leq h \leq 14$

$-33 \leq k \leq 45$

$-24 \leq l \leq 23$

16537(0.0317)

16537 / 0 / 874

1.026

0.0310

0.0698

$1.669,-0.809$

$\left.{ }^{\mathrm{a}} R 1=\sum|| \mathrm{F}_{\mathrm{o}}-\left|\mathrm{F}_{c}\right|\left|/ \sum\right| \mathrm{F}_{\mathrm{o}} \mid ; \mathrm{b}_{w} R 2=\left\{\sum\left[w\left(F_{o}{ }^{2}-F_{c}{ }^{2}\right)^{2}\right] / \sum w\left(F_{o}^{2}\right)^{2}\right]\right\}^{1 / 2}$ 
Table S3. Crystallographic Data for $\left[\mathrm{Re}(\mathrm{dmpp})_{3}\right] \mathrm{B}\left(\mathrm{C}_{6} \mathrm{~F}_{5}\right)_{4} \cdot \mathrm{CH}_{2} \mathrm{Cl}_{2}(\mathbf{5})$ and $\left[\mathrm{Re}(\mathrm{dmpb})_{3}\right]$ $\mathrm{B}\left(\mathrm{C}_{6} \mathrm{~F}_{5}\right)_{4}(7)$.

5

$\overline{\text { CCDC }}$

Empirical formula

Color

Formula weight

Crystal dimensions

Crystal system, Space group

$a(\AA)$

$b(\AA)$

$c(\AA)$

$\alpha\left(^{\circ}\right)$

$\beta\left(^{\circ}\right)$

$\gamma\left({ }^{\circ}\right)$

$V\left(\AA^{3}\right)$

$Z$

$\rho_{\text {calcd }}\left(\mathrm{g} \mathrm{cm}^{-1}\right)$

$\mu\left(\mathrm{mm}^{-1}\right)$

$2 \theta$ limit $\left({ }^{\circ}\right)$

Index ranges

Independent reflections $\left(R_{\text {int }}\right)$

Data/restraints/parameters

Goodness-of-fit

$R 1[I>2 \sigma(I)]^{a}$

$w R 2[I>2 \sigma(I)]^{\mathrm{b}}$

Largest peak, hole $\left(\mathrm{e}^{-3}\right)$
$\mathrm{C}_{45} \mathrm{H}_{64} \mathrm{BF}_{20} \mathrm{P}_{6} \mathrm{Re}$

Colorless

1367.79

$0.25 \times 0.16 \times 0.05$

Triclinic, $P \overline{1}$

$17.2940(2)$

17.6696(3)

27.3846(4)

72.514(1)

78.494(1)

$77.689(1)$

$4228.39(13)$

6

1.766

2.656

$1.58-33.73$

$-26 \leq h \leq 27$

$-27 \leq k \leq 27$

$-38 \leq l \leq 42$

$61018(0.0390)$

61018 / 0 / 1972

1.047

0.0829

0.2221

$9.734,-3.000$
7

$\mathrm{C}_{54} \mathrm{H}_{48} \mathrm{BF}_{20} \mathrm{P}_{6} \mathrm{Re}$

Colorless

1459.75

$0.47 \times 0.32 \times 0.13$

monoclinic, $C c$

16.2261(2)

22.9605(2)

$32.0279(4)$

90

102.157(1)

90

11664.7(2)

8

1.662

2.349

$1.77-33.73$

$-25 \leq h \leq 21$

$-35 \leq k \leq 27$

$-40 \leq l \leq 50$

35983 (0.0586)

35983 / 16 / 1478

1.011

0.0484

0.1196

5.191, -2.188

$\left.{ }^{\mathrm{a}} R 1=\sum|| \mathrm{F}_{\mathrm{o}}-\left|\mathrm{F}_{c}\right|\left|/ \sum\right| \mathrm{F}_{\mathrm{o}} \mid ; \mathrm{b}_{w} R 2=\left\{\sum\left[w\left(F_{o}{ }^{2}-F_{c}{ }^{2}\right)^{2}\right] / \sum w\left(F_{o}^{2}\right)^{2}\right]\right\}^{1 / 2}$ 
Table S4. Crystallographic Data for $\left[\mathrm{Re}(\mathrm{dmpe})_{3}\right]\left(\mathrm{B}\left(\mathrm{C}_{6} \mathrm{~F}_{5}\right)_{4}\right)_{2} \cdot 3 \mathrm{MeCN}(\mathbf{8})$ and $\left[\operatorname{Re}(\mathrm{depe})_{3}\right]$ $\left(\mathrm{B}\left(\mathrm{C}_{6} \mathrm{~F}_{5}\right)_{4}\right)_{2} \cdot 2 \mathrm{MeOH}(\mathbf{9})$.

\section{8} 9

\begin{tabular}{|c|c|c|}
\hline \multicolumn{3}{|l|}{$\overline{\mathrm{CCDC}}$} \\
\hline Empirical formula & $\mathrm{C}_{72} \mathrm{H}_{57} \mathrm{~B}_{2} \mathrm{~F}_{40} \mathrm{~N}_{3} \mathrm{P}_{6} \mathrm{Re}$ & $\mathrm{C}_{80} \mathrm{H}_{80} \mathrm{~B}_{2} \mathrm{~F}_{40} \mathrm{O}_{2} \mathrm{P}_{6} \mathrm{Re}$ \\
\hline Formula weight & 2117.85 & 2227.08 \\
\hline Color & Red & Pink-red \\
\hline Crystal dimensions & $0.31 \times 0.21 \times 0.14$ & $0.27 \times 0.20 \times 0.12$ \\
\hline Crystal system, Space group & Triclinic, $P \overline{1}$ & Monoclinic, $P 2_{1} / c$ \\
\hline$a(\AA)$ & $12.3644(1)$ & $24.4989(6)$ \\
\hline$b(\AA)$ & $15.5167(1)$ & $15.7767(4)$ \\
\hline$c(\AA)$ & $21.6151(2)$ & $23.3647(5)$ \\
\hline$\alpha\left({ }^{\circ}\right)$ & $78.654(1)$ & 90 \\
\hline$\beta\left(^{\circ}\right)$ & $81.365(1)$ & $92.819(1)$ \\
\hline$\gamma\left({ }^{\circ}\right)$ & $80.171(1)$ & 90 \\
\hline$V\left(\AA^{3}\right)$ & $3976.97(6)$ & $9019.8(4)$ \\
\hline$Z$ & 2 & 4 \\
\hline$\rho_{\text {calcd }}\left(\mathrm{g} \mathrm{cm}^{-1}\right)$ & 1.769 & 1.640 \\
\hline$\mu\left(\mathrm{mm}^{-1}\right)$ & 1.788 & 1.582 \\
\hline $2 \theta \operatorname{limit}\left({ }^{\circ}\right)$ & $1.80-28.28$ & $1.56-28.70$ \\
\hline \multirow[t]{3}{*}{ Index ranges } & $-16 \leq h \leq 16$ & $-29 \leq h \leq 32$ \\
\hline & $-20 \leq k \leq 20$ & $-21 \leq k \leq 16$ \\
\hline & $-28 \leq l \leq 28$ & $-31 \leq l \leq 31$ \\
\hline Independent reflections $\left(R_{\text {int }}\right)$ & $19474(0.0342)$ & $23030(0.0569)$ \\
\hline Data/restraints/parameters & 19474 / 0 / 1117 & 23030 / 0 / 1178 \\
\hline Goodness-of-fit & 1.028 & 1.030 \\
\hline$R 1[I>2 \sigma(I)]^{a}$ & 0.0397 & 0.0489 \\
\hline$w R 2[I>2 \sigma(I)]^{\mathrm{b}}$ & 0.0903 & 0.1241 \\
\hline Largest peak, hole $\left(\mathrm{e} \AA^{-3}\right)$ & $3.208,-1.440$ & $1.969,-0.825$ \\
\hline
\end{tabular}


Table S5. Crystallographic Data for $\left[\mathrm{Re}(\mathrm{dmpm})_{3}\right]\left(\mathrm{B}\left(\mathrm{C}_{6} \mathrm{~F}_{5}\right)_{4}\right)_{2} \cdot \mathrm{CH}_{2} \mathrm{Cl}_{2}(\mathbf{1 0})$ and $\left[\mathrm{Re}\left(\mathrm{Me}_{2} \mathrm{PCH}_{2} \mathrm{PPh}_{2}\right)_{3}\right]_{2}\left(\mathrm{PF}_{6}\right)_{3}(\mathbf{1 1})$.

1011

$\overline{\mathrm{CCDC}}$

Empirical formula $\quad \mathrm{C}_{64} \mathrm{H}_{44} \mathrm{~B}_{2} \mathrm{Cl}_{2} \mathrm{~F}_{40} \mathrm{P}_{6} \mathrm{Re} \quad \mathrm{C}_{90} \mathrm{H}_{108} \mathrm{~F}_{18} \mathrm{P}_{15} \mathrm{Re}_{2}$

Color

Pink-red

Pink

Formula weight

2037.53

2368.71

Crystal dimensions

$0.53 \times 0.44 \times 0.07$

$0.40 \times 0.37 \times 0.33$

Crystal system, Space group

Monoclinic, $P 21 / c$

Cubic, $\operatorname{Pa} \overline{3}$

$a(\AA)$

11.8283(2)

20.9641(6)

$b(\AA)$

27.5978(5)

20.9641(6)

$c(\AA)$

22.4523(4)

20.9641(6)

$\alpha\left(^{\circ}\right)$

90

90

$\beta\left(^{\circ}\right)$

99.528(1)

90

$\gamma\left({ }^{\circ}\right)$

90

90

$V\left(\AA^{3}\right)$

7228.1(2)

9213.6(5)

$Z$

4

4

$\rho_{\text {calcd }}\left(\mathrm{g} \mathrm{cm}^{-1}\right)$

1.872

1.708

$\mu\left(\mathrm{mm}^{-1}\right)$

2.034

2.969

$2 \theta \operatorname{limit}\left({ }^{\circ}\right)$

$1.74-31.51$

$2.38-28.69$

Index ranges

$-17 \leq h \leq 17$

$-28 \leq h \leq 28$

$-40 \leq k \leq 40$

$-26 \leq k \leq 27$

$-32 \leq l \leq 32$

$-28 \leq l \leq 28$

Independent reflections $\left(R_{i n t}\right)$

23815 (0.0708)

$3964(0.0327)$

Data/restraints/parameters

23815 / 0 / 1064

3964 / 0 / 235

Goodness-of-fit

1.040

1.138

$R 1[I>2 \sigma(I)]^{a}$

0.0708

0.0306

$w R 2[I>2 \sigma(I)]^{\mathrm{b}}$

0.1868

0.0713

Largest peak, hole $\left(\mathrm{e}^{-3}\right)$

$5.069,-2.533$

1.226, -0.876 
Table S6. Crystallographic Data for $\left[\mathrm{Re}(\mathrm{dmpb})_{3}\right]\left(\mathrm{SbF}_{6}\right)_{2} \cdot \mathrm{PhCl}(\mathbf{1 4})$

\section{4}

\begin{tabular}{|c|c|}
\hline$\overline{\mathrm{CCDC}}$ & \\
\hline Empirical formula & $\mathrm{C}_{36} \mathrm{H}_{53} \mathrm{ClF}_{12} \mathrm{P}_{6} \mathrm{ReSb}_{2}$ \\
\hline Color & red \\
\hline Formula weight & 1364.75 \\
\hline Crystal dimensions & $0.30 \times 0.26 \times 0.09 \mathrm{~mm}^{3}$ \\
\hline Crystal system, space group & Monoclinic, $P 2_{1}$ \\
\hline$a(\AA)$ & $13.4084(4)$ \\
\hline$b(\AA)$ & $21.1279(6)$ \\
\hline$c(\AA)$ & $17.1012(6)$ \\
\hline$\alpha\left(^{\circ}\right)$ & 90 \\
\hline$\beta\left(^{\circ}\right)$ & $104.330(2)$ \\
\hline$\gamma\left({ }^{\circ}\right)$ & 90 \\
\hline$V\left(\AA^{3}\right)$ & $4693.9(3)$ \\
\hline$Z$ & 4 \\
\hline$\rho_{\text {calcd }}\left(\mathrm{g} \mathrm{cm}^{-1}\right)$ & 1.931 \\
\hline$\mu\left(\mathrm{mm}^{-1}\right)$ & 4.051 \\
\hline $2 \theta \operatorname{limit}\left({ }^{\circ}\right)$ & $1.74-33.73$ \\
\hline Index ranges & $-19 \leq h \leq 19$ \\
\hline & $-28 \leq k \leq 31$ \\
\hline & $-19 \leq l \leq 25$ \\
\hline Independent reflections $\left(R_{\text {int }}\right)$ & $29384(0.0464)$ \\
\hline Data/restraints/parameters & 29384 / 16 / 1045 \\
\hline Goodness-of-fit & 0.985 \\
\hline$R 1[I>2 \sigma(I)]^{a}$ & 0.0387 \\
\hline$w R 2[I>2 \sigma(I)]^{\mathrm{b}}$ & 0.0706 \\
\hline Largest peak, hole $\left(\mathrm{e}^{-3}\right)$ & $2.035,-0.891$ \\
\hline
\end{tabular}


Table S7. Crystallographic Data for $\left[\operatorname{Re}(\mathrm{dmpm})_{3}(\mathrm{MeCN})\right]\left(\mathrm{SbF}_{6}\right)_{3}(\mathbf{1 5})$

15

\begin{tabular}{|c|c|}
\hline$\overline{\mathrm{CCDC}}$ & \\
\hline Empirical formula & $\mathrm{C}_{17} \mathrm{H}_{45} \mathrm{~F}_{18} \mathrm{NP}_{6} \mathrm{ReSb}_{3}$ \\
\hline Color & orange \\
\hline Formula weight & 1342.81 \\
\hline Crystal dimensions & $0.21 \times 0.20 \times 0.18 \mathrm{~mm}^{3}$ \\
\hline Crystal system, space group & Monoclinic, $C 2 / \mathrm{c}$ \\
\hline$a(\AA)$ & $19.3297(2)$ \\
\hline$b(\AA)$ & $11.0763(1)$ \\
\hline$c(\AA)$ & $7.7264(5)$ \\
\hline$\beta\left(^{\circ}\right)$ & $104.400(1)$ \\
\hline$V\left(\AA^{3}\right)$ & $7823.52(15)$ \\
\hline$Z$ & 8 \\
\hline$\rho_{\text {calcd }}\left(\mathrm{g} \mathrm{cm}^{-1}\right)$ & 2.280 \\
\hline$\mu\left(\mathrm{mm}^{-1}\right)$ & 5.480 \\
\hline $2 \theta \operatorname{limit}\left({ }^{\circ}\right)$ & 2.28 to $33.73^{\circ}$ \\
\hline Index ranges & $-28 \leq h \leq 30$ \\
\hline & $-14 \leq k \leq 17$ \\
\hline & $-58 \leq l \leq 54$ \\
\hline Independent reflections $\left(R_{\text {int }}\right)$ & $15524(0.0421)$ \\
\hline Data/restraints/parameters & $15524 / 0$ / 445 \\
\hline Goodness-of-fit & 1.106 \\
\hline$R 1[I>2 \sigma(I)]^{a}$ & 0.0567 \\
\hline$w R 2[I>2 \sigma(I)]^{\mathrm{b}}$ & 0.1211 \\
\hline Largest peak, hole $\left(\mathrm{e}^{-3}\right)$ & $3.272,-2.725$ \\
\hline
\end{tabular}


Table S8. Selected Bond Distances $(\AA)$ and Angles $\left({ }^{\circ}\right)$ for $\left[\operatorname{Re}(d m p e)_{3}\right] \mathrm{B}\left(\mathrm{C}_{6} \mathrm{~F}_{5}\right)_{4} \cdot \mathrm{CH}_{2} \mathrm{Cl}_{2}(\mathbf{1})$.

$\begin{array}{llll}\operatorname{Re}(1)-\mathrm{P}(3) & 2.3719(7) & \operatorname{Re}(1)-\mathrm{P}(6) & 2.3773(7) \\ \operatorname{Re}(1)-\mathrm{P}(4) & 2.3785(9) & \operatorname{Re}(1)-\mathrm{P}(1) & 2.3854(7) \\ \operatorname{Re}(1)-\mathrm{P}(2) & 2.3859(7) & \operatorname{Re}(1)-\mathrm{P}(5) & 2.3889(9) \\ & & & \\ \mathrm{P}(3)-\operatorname{Re}(1)-\mathrm{P}(6) & 93.94(3) & \mathrm{P}(3)-\operatorname{Re}(1)-\mathrm{P}(4) & 80.84(3) \\ \mathrm{P}(6)-\operatorname{Re}(1)-\mathrm{P}(4) & 90.72(3) & \mathrm{P}(3)-\operatorname{Re}(1)-\mathrm{P}(1) & 91.33(3) \\ \mathrm{P}(6)-\operatorname{Re}(1)-\mathrm{P}(1) & 173.89(3) & \mathrm{P}(4)-\operatorname{Re}(1)-\mathrm{P}(1) & 93.16(3) \\ \mathrm{P}(3)-\operatorname{Re}(1)-\mathrm{P}(2) & 172.20(3) & \mathrm{P}(6)-\operatorname{Re}(1)-\mathrm{P}(2) & 92.13(3) \\ \mathrm{P}(4)-\operatorname{Re}(1)-\mathrm{P}(2) & 94.19(3) & \mathrm{P}(1)-\operatorname{Re}(1)-\mathrm{P}(2) & 82.89(3) \\ \mathrm{P}(3)-\operatorname{Re}(1)-\mathrm{P}(5) & 92.46(4) & \mathrm{P}(6)-\operatorname{Re}(1)-\mathrm{P}(5) & 83.46(4) \\ \mathrm{P}(4)-\operatorname{Re}(1)-\mathrm{P}(5) & 170.83(4) & \mathrm{P}(1)-\operatorname{Re}(1)-\mathrm{P}(5) & 93.24(4) \\ \mathrm{P}(2)-\operatorname{Re}(1)-\mathrm{P}(5) & 93.10(4) & & \end{array}$


Table S9. Selected Bond Distances $(\AA)$ and Angles $\left({ }^{\circ}\right)$ for $\left[\operatorname{Re}(\text { depe })_{3}\right] \mathrm{B}\left(\mathrm{C}_{6} \mathrm{~F}_{5}\right)_{4}(\mathbf{2})$.

$\begin{array}{llll}\operatorname{Re}(1)-\mathrm{P}(5) & 2.3955(5) & \operatorname{Re}(1)-\mathrm{P}(6) & 2.3966(6) \\ \operatorname{Re}(1)-\mathrm{P}(4) & 2.4034(6) & \operatorname{Re}(1)-\mathrm{P}(2) & 2.4069(6) \\ \operatorname{Re}(1)-\mathrm{P}(3) & 2.4132(6) & \operatorname{Re}(1)-\mathrm{P}(1) & 2.4139(5) \\ & & & \\ \mathrm{P}(5)-\operatorname{Re}(1)-\mathrm{P}(6) & 81.35(2) & \mathrm{P}(5)-\operatorname{Re}(1)-\mathrm{P}(4) & 94.91(2) \\ \mathrm{P}(6)-\operatorname{Re}(1)-\mathrm{P}(4) & 90.09(2) & \mathrm{P}(5)-\operatorname{Re}(1)-\mathrm{P}(2) & 90.06(2) \\ \mathrm{P}(6)-\operatorname{Re}(1)-\mathrm{P}(2) & 94.66(2) & \mathrm{P}(4)-\operatorname{Re}(1)-\mathrm{P}(2) & 173.59(2) \\ \mathrm{P}(5)-\operatorname{Re}(1)-\mathrm{P}(3) & 93.10(2) & \mathrm{P}(6)-\operatorname{Re}(1)-\mathrm{P}(3) & 168.81(2) \\ \mathrm{P}(4)-\operatorname{Re}(1)-\mathrm{P}(3) & 80.66(2) & \mathrm{P}(2)-\operatorname{Re}(1)-\mathrm{P}(3) & 95.05(2) \\ \mathrm{P}(5)-\operatorname{Re}(1)-\mathrm{P}(1) & 169.30(2) & \mathrm{P}(6)-\operatorname{Re}(1)-\mathrm{P}(1) & 93.80(2) \\ \mathrm{P}(4)-\operatorname{Re}(1)-\mathrm{P}(1) & 94.63(2) & \mathrm{P}(2)-\operatorname{Re}(1)-\mathrm{P}(1) & 80.78(2)\end{array}$

$\mathrm{P}(3)-\operatorname{Re}(1)-\mathrm{P}(1) \quad 93.21(2)$ 
Table S10. Selected Bond Distances $(\AA)$ and Angles $\left({ }^{\circ}\right)$ for $\left[\operatorname{Re}(\mathrm{dmpm})_{3}\right] \mathrm{B}\left(\mathrm{C}_{6} \mathrm{~F}_{5}\right)_{4}(3)$.

$\begin{array}{llll}\operatorname{Re}(1)-\mathrm{P}(1) & 2.3587(7) & \operatorname{Re}(1)-\mathrm{P}(2) & 2.3515(9) \\ \operatorname{Re}(1)-\mathrm{P}(3) & 2.4482(11) & \operatorname{Re}(1)-\mathrm{P}(3 \mathrm{~A}) & 2.3963(18) \\ \operatorname{Re}(1)-\mathrm{P}(4) & 2.373(5) & \operatorname{Re}(1)-\mathrm{P}(4 \mathrm{~A}) & 2.337(6) \\ \operatorname{Re}(1)-\mathrm{P}(5) & 2.359(3) & \operatorname{Re}(1)-\mathrm{P}(5 \mathrm{~A}) & 2.381(4) \\ \operatorname{Re}(1)-\mathrm{P}(6) & 2.3366(12) & \operatorname{Re}(1)-\mathrm{P}(6 \mathrm{~A}) & 2.2828(15) \\ & & & \\ \mathrm{P}(1)-\operatorname{Re}(1)-\mathrm{P}(2) & 69.08(3) & \mathrm{P}(1)-\operatorname{Re}(1)-\mathrm{P}(3) & 88.97(3) \\ \mathrm{P}(1)-\operatorname{Re}(1)-\mathrm{P}(5) & 103.99(7) & \mathrm{P}(1)-\operatorname{Re}(1)-\mathrm{P}(6) & 166.12(4) \\ \mathrm{P}(2)-\operatorname{Re}(1)-\mathrm{P}(3) & 155.35(4) & \mathrm{P}(2)-\operatorname{Re}(1)-\mathrm{P}(4) & 100.77(15) \\ \mathrm{P}(2)-\operatorname{Re}(1)-\mathrm{P}(5) & 101.85(7) & \mathrm{P}(2)-\operatorname{Re}(1)-\mathrm{P}(6) & 99.72(5) \\ \mathrm{P}(3)-\operatorname{Re}(1)-\mathrm{P}(4) & 68.05(15) & \mathrm{P}(3 \mathrm{~A})-\operatorname{Re}(1)-\mathrm{P}(4 \mathrm{~A}) & 71.5(2) \\ \mathrm{P}(3)-\operatorname{Re}(1)-\mathrm{P}(5) & 94.06(7) & \mathrm{P}(3 \mathrm{~A})-\operatorname{Re}(1)-\mathrm{P}(5 \mathrm{~A}) & 98.23(13) \\ \mathrm{P}(3)-\operatorname{Re}(1)-\mathrm{P}(6) & 103.47(5) & \mathrm{P}(3 \mathrm{~A})-\operatorname{Re}(1)-\mathrm{P}(6 \mathrm{~A}) & 103.58(7) \\ \mathrm{P}(4)-\operatorname{Re}(1)-\mathrm{P}(5) & 155.59(16) & \mathrm{P}(4 \mathrm{~A})-\operatorname{Re}(1)-\mathrm{P}(5 \mathrm{~A}) & 162.7(2) \\ \mathrm{P}(4)-\operatorname{Re}(1)-\mathrm{P}(6) & 97.75(13) & \mathrm{P}(4 \mathrm{~A})-\operatorname{Re}(1)-\mathrm{P}(6 \mathrm{~A}) & 96.6(2) \\ \mathrm{P}(5)-\operatorname{Re}(1)-\mathrm{P}(6) & 69.46(7) & \mathrm{P}(5 \mathrm{~A})-\operatorname{Re}(1)-\mathrm{P}(6 \mathrm{~A}) & 71.76(10) \\ \mathrm{P}(1)-\operatorname{Re}(1)-\mathrm{P}(4) & 92.56(12) & & \end{array}$


Table S11. Selected Bond Distances $(\AA)$ and Angles $\left({ }^{\circ}\right)$ for $f a c-\left[\operatorname{Re}\left(\mathrm{Me}_{2} \mathrm{PCH}_{2} \mathrm{PPh}_{2}\right)_{3}\right]$ $\mathrm{B}\left(\mathrm{C}_{6} \mathrm{~F}_{5}\right)_{4}(\mathbf{4})$.

$\begin{array}{llll}\operatorname{Re}(1)-\mathrm{P}(3) & 2.3683(7) & \operatorname{Re}(1)-\mathrm{P}(5) & 2.3765(7) \\ \operatorname{Re}(1)-\mathrm{P}(1) & 2.3821(7) & \operatorname{Re}(1)-\mathrm{P}(4) & 2.4001(7) \\ \operatorname{Re}(1)-\mathrm{P}(2) & 2.4056(7) & \operatorname{Re}(1)-\mathrm{P}(6) & 2.4506(7) \\ & & & \\ \mathrm{P}(3)-\operatorname{Re}(1)-\mathrm{P}(5) & 97.11(2) & \mathrm{P}(3)-\operatorname{Re}(1)-\mathrm{P}(1) & 91.45(2) \\ \mathrm{P}(5)-\operatorname{Re}(1)-\mathrm{P}(1) & 93.82(3) & \mathrm{P}(3)-\operatorname{Re}(1)-\mathrm{P}(4) & 68.75(2) \\ \mathrm{P}(5)-\operatorname{Re}(1)-\mathrm{P}(4) & 99.23(2) & \mathrm{P}(1)-\operatorname{Re}(1)-\mathrm{P}(4) & 157.39(2) \\ \mathrm{P}(3)-\operatorname{Re}(1)-\mathrm{P}(2) & 91.66(2) & \mathrm{P}(5)-\operatorname{Re}(1)-\mathrm{P}(2) & 160.62(2) \\ \mathrm{P}(1)-\operatorname{Re}(1)-\mathrm{P}(2) & 68.62(2) & \mathrm{P}(4)-\operatorname{Re}(1)-\mathrm{P}(2) & 100.05(2) \\ \mathrm{P}(3)-\operatorname{Re}(1)-\mathrm{P}(6) & 158.68(2) & \mathrm{P}(5)-\operatorname{Re}(1)-\mathrm{P}(6) & 68.07(2) \\ \mathrm{P}(1)-\operatorname{Re}(1)-\mathrm{P}(6) & 104.35(2) & \mathrm{P}(4)-\operatorname{Re}(1)-\mathrm{P}(6) & 97.65(2) \\ \mathrm{P}(2)-\operatorname{Re}(1)-\mathrm{P}(6) & 107.27(2) & & \end{array}$


Table S12. Selected Bond Distances $(\AA)$ and Angles $\left({ }^{\circ}\right)$ for $\left[\operatorname{Re}(\mathrm{dmpp})_{3}\right] \mathrm{B}\left(\mathrm{C}_{6} \mathrm{~F}_{5}\right)_{4}(\mathbf{5})$.

$\begin{array}{llll}\operatorname{Re}(1)-\mathrm{P}(1) & 2.477(3) & \mathrm{Re}(2)-\mathrm{P}(7) & 2.395(3) \\ \operatorname{Re}(1)-\mathrm{P}(2) & 2.501(3) & \mathrm{Re}(2)-\mathrm{P}(8) & 2.403(2) \\ \operatorname{Re}(1)-\mathrm{P}(3) & 2.338(3) & \operatorname{Re}(2)-\mathrm{P}(9) & 2.402(2) \\ \operatorname{Re}(1)-\mathrm{P}(4) & 2.447(3) & \operatorname{Re}(2)-\mathrm{P}(10) & 2.422(3) \\ \operatorname{Re}(1)-\mathrm{P}(5) & 2.357(4) & \mathrm{Re}(2)-\mathrm{P}(11) & 2.464(3) \\ \operatorname{Re}(1)-\mathrm{P}(6) & 2.405(3) & \mathrm{Re}(2)-\mathrm{P}(12) & 2.410(2) \\ & & & \\ \mathrm{P}(1)-\operatorname{Re}(1)-\mathrm{P}(2) & 86.81(13) & \mathrm{P}(7)-\operatorname{Re}(2)-\mathrm{P}(8) & 93.31(11) \\ \mathrm{P}(1)-\operatorname{Re}(1)-\mathrm{P}(3) & 86.11(13) & \mathrm{P}(7)-\operatorname{Re}(2)-\mathrm{P}(9) & 88.44(12) \\ \mathrm{P}(1)-\operatorname{Re}(1)-\mathrm{P}(4) & 87.56(12) & \mathrm{P}(7)-\operatorname{Re}(2)-\mathrm{P}(10) & 86.86(12) \\ \mathrm{P}(1)-\operatorname{Re}(1)-\mathrm{P}(5) & 178.20(11) & \mathrm{P}(7)-\operatorname{Re}(2)-\mathrm{P}(11) & 97.59(12) \\ \mathrm{P}(1)-\operatorname{Re}(1)-\mathrm{P}(6) & 90.87(12) & \mathrm{P}(7)-\operatorname{Re}(2)-\mathrm{P}(12) & 174.82(11) \\ \mathrm{P}(2)-\operatorname{Re}(1)-\mathrm{P}(3) & 170.91(14) & \mathrm{P}(8)-\operatorname{Re}(2)-\mathrm{P}(9) & 89.18(9) \\ \mathrm{P}(2)-\operatorname{Re}(1)-\mathrm{P}(4) & 83.41(13) & \mathrm{P}(8)-\operatorname{Re}(2)-\mathrm{P}(10) & 179.67(14) \\ \mathrm{P}(2)-\operatorname{Re}(1)-\mathrm{P}(5) & 92.59(13) & \mathrm{P}(8)-\operatorname{Re}(2)-\mathrm{P}(11) & 93.36(10) \\ \mathrm{P}(2)-\operatorname{Re}(1)-\mathrm{P}(6) & 88.98(13) & \mathrm{P}(8)-\operatorname{Re}(2)-\mathrm{P}(12) & 91.87(10) \\ \mathrm{P}(3)-\operatorname{Re}(1)-\mathrm{P}(4) & 90.60(15) & \mathrm{P}(9)-\operatorname{Re}(2)-\mathrm{P}(10) & 91.10(11) \\ \mathrm{P}(3)-\operatorname{Re}(1)-\mathrm{P}(5) & 94.65(14) & \mathrm{P}(9)-\operatorname{Re}(2)-\mathrm{P}(11) & 173.30(9) \\ \mathrm{P}(4)-\operatorname{Re}(1)-\mathrm{P}(5) & 94.06(13) & \mathrm{P}(10)-\operatorname{Re}(2)-\mathrm{P}(11) & 86.34(11) \\ \mathrm{P}(3)-\operatorname{Re}(1)-\mathrm{P}(6) & 96.82(15) & \mathrm{P}(9)-\operatorname{Re}(2)-\mathrm{P}(12) & 91.49(8) \\ \mathrm{P}(4)-\operatorname{Re}(1)-\mathrm{P}(6) & 172.30(15) & \mathrm{P}(10)-\operatorname{Re}(2)-\mathrm{P}(12) & 87.97(10) \\ \mathrm{P}(5)-\operatorname{Re}(1)-\mathrm{P}(6) & 87.42(13) & \mathrm{P}(11)-\operatorname{Re}(2)-\mathrm{P}(12) & 82.24(9)\end{array}$


Table S13. Selected Bond Distances $(\AA)$ and Angles $\left({ }^{\circ}\right)$ for $\left[\operatorname{Re}(\mathrm{dmpb})_{3}\right] \mathrm{B}\left(\mathrm{C}_{6} \mathrm{~F}_{5}\right)_{4}(7)$.

$\begin{array}{llll}\operatorname{Re}(1)-\mathrm{P}(1) & 2.3647(14) & \mathrm{Re}(2)-\mathrm{P}(7) & 2.3636(16) \\ \operatorname{Re}(1)-\mathrm{P}(2) & 2.3693(17) & \mathrm{Re}(2)-\mathrm{P}(8) & 2.3659(14) \\ \operatorname{Re}(1)-\mathrm{P}(3) & 2.3789(15) & \mathrm{Re}(2)-\mathrm{P}(9) & 2.3820(15) \\ \operatorname{Re}(1)-\mathrm{P}(4) & 2.3799(14) & \mathrm{Re}(2)-\mathrm{P}(10) & 2.3827(15) \\ \operatorname{Re}(1)-\mathrm{P}(5) & 2.3709(16) & \mathrm{Re}(2)-\mathrm{P}(11) & 2.3813(14) \\ \operatorname{Re}(1)-\mathrm{P}(6) & 2.3689(15) & \mathrm{Re}(2)-\mathrm{P}(12) & 2.3624(15) \\ & & & \\ \mathrm{P}(1)-\operatorname{Re}(1)-\mathrm{P}(2) & 81.92(5) & \mathrm{P}(7)-\operatorname{Re}(2)-\mathrm{P}(8) & 82.47(5) \\ \mathrm{P}(1)-\operatorname{Re}(1)-\mathrm{P}(3) & 90.45(5) & \mathrm{P}(7)-\operatorname{Re}(2)-\mathrm{P}(9) & 92.95(5) \\ \mathrm{P}(1)-\operatorname{Re}(1)-\mathrm{P}(4) & 169.66(5) & \mathrm{P}(7)-\operatorname{Re}(2)-\mathrm{P}(10) & 170.17(5) \\ \mathrm{P}(1)-\operatorname{Re}(1)-\mathrm{P}(5) & 91.95(5) & \mathrm{P}(7)-\operatorname{Re}(2)-\mathrm{P}(11) & 90.09(5) \\ \mathrm{P}(1)-\operatorname{Re}(1)-\mathrm{P}(6) & 95.17(5) & \mathrm{P}(7)-\operatorname{Re}(2)-\mathrm{P}(12) & 92.18(5) \\ \mathrm{P}(2)-\operatorname{Re}(1)-\mathrm{P}(3) & 92.61(6) & \mathrm{P}(8)-\operatorname{Re}(2)-\mathrm{P}(9) & 91.51(5) \\ \mathrm{P}(2)-\operatorname{Re}(1)-\mathrm{P}(4) & 90.94(6) & \mathrm{P}(8)-\operatorname{Re}(2)-\mathrm{P}(10) & 89.32(5) \\ \mathrm{P}(2)-\operatorname{Re}(1)-\mathrm{P}(5) & 171.75(5) & \mathrm{P}(8)-\operatorname{Re}(2)-\mathrm{P}(11) & 170.87(5) \\ \mathrm{P}(2)-\operatorname{Re}(1)-\mathrm{P}(6) & 92.63(6) & \mathrm{P}(8)-\operatorname{Re}(2)-\mathrm{P}(12) & 93.00(5) \\ \mathrm{P}(3)-\operatorname{Re}(1)-\mathrm{P}(4) & 82.38(5) & \mathrm{P}(9)-\operatorname{Re}(2)-\mathrm{P}(10) & 81.83(5) \\ \mathrm{P}(3)-\operatorname{Re}(1)-\mathrm{P}(5) & 92.95(5) & \mathrm{P}(9)-\operatorname{Re}(2)-\mathrm{P}(11) & 94.16(5) \\ \mathrm{P}(3)-\operatorname{Re}(1)-\mathrm{P}(6) & 172.80(5) & \mathrm{P}(9)-\operatorname{Re}(2)-\mathrm{P}(12) & 173.57(5) \\ \mathrm{P}(4)-\operatorname{Re}(1)-\mathrm{P}(5) & 95.83(5) & \mathrm{P}(10)-\operatorname{Re}(2)-\mathrm{P}(11) & 98.56(5) \\ \mathrm{P}(4)-\operatorname{Re}(1)-\mathrm{P}(6) & 92.60(5) & \mathrm{P}(10)-\operatorname{Re}(2)-\mathrm{P}(12) & 93.64(5) \\ \mathrm{P}(5)-\operatorname{Re}(1)-\mathrm{P}(6) & 82.38(5) & \mathrm{P}(11)-\operatorname{Re}(2)-\mathrm{P}(12) & 81.96(5)\end{array}$


Table S14. Selected Bond Distances $(\AA)$ and Angles $\left({ }^{\circ}\right)$ for $\left[\operatorname{Re}(\mathrm{dmpe})_{3}\right]\left(\mathrm{B}\left(\mathrm{C}_{6} \mathrm{~F}_{5}\right)_{4}\right)_{2}$. $3 \mathrm{MeCN}(\mathbf{8})$.

$\begin{array}{llll}\operatorname{Re}(1)-\mathrm{P}(1) & 2.4690(10) & \operatorname{Re}(1)-\mathrm{P}(2) & 2.4440(9) \\ \operatorname{Re}(1)-\mathrm{P}(3) & 2.4440(9) & \operatorname{Re}(1)-\mathrm{P}(4) & 2.4423(11) \\ \operatorname{Re}(1)-\mathrm{P}(5) & 2.4453(10) & \operatorname{Re}(1)-\mathrm{P}(6) & 2.4410(9) \\ & & & \\ \mathrm{P}(6)-\operatorname{Re}(1)-\mathrm{P}(4) & 94.31(4) & \mathrm{P}(6)-\operatorname{Re}(1)-\mathrm{P}(2) & 93.94(3) \\ \mathrm{P}(4)-\operatorname{Re}(1)-\mathrm{P}(2) & 91.66(4) & \mathrm{P}(6)-\operatorname{Re}(1)-\mathrm{P}(3) & 171.30(3) \\ \mathrm{P}(4)-\operatorname{Re}(1)-\mathrm{P}(3) & 81.57(4) & \mathrm{P}(2)-\operatorname{Re}(1)-\mathrm{P}(3) & 93.83(3) \\ \mathrm{P}(6)-\operatorname{Re}(1)-\mathrm{P}(5) & 80.96(3) & \mathrm{P}(4)-\operatorname{Re}(1)-\mathrm{P}(5) & 93.14(4) \\ \mathrm{P}(2)-\operatorname{Re}(1)-\mathrm{P}(5) & 173.24(3) & \mathrm{P}(3)-\operatorname{Re}(1)-\mathrm{P}(5) & 91.58(4) \\ \mathrm{P}(6)-\operatorname{Re}(1)-\mathrm{P}(1) & 91.26(3) & \mathrm{P}(4)-\operatorname{Re}(1)-\mathrm{P}(1) & 170.26(4) \\ \mathrm{P}(2)-\operatorname{Re}(1)-\mathrm{P}(1) & 79.97(3) & \mathrm{P}(3)-\operatorname{Re}(1)-\mathrm{P}(1) & 93.93(3) \\ \mathrm{P}(5)-\operatorname{Re}(1)-\mathrm{P}(1) & 95.63(3) & & \end{array}$


Table S15. Selected Bond Distances $(\AA)$ and Angles $\left({ }^{\circ}\right)$ for $\left[\operatorname{Re}(\text { depe })_{3}\right]\left(B\left(\mathrm{C}_{6} \mathrm{~F}_{5}\right)_{4}\right)_{2}$. $2 \mathrm{MeOH}(9)$.

$\begin{array}{llll}\operatorname{Re}(1)-\mathrm{P}(1) & 2.4714(11) & \operatorname{Re}(1)-\mathrm{P}(2) & 2.4632(12) \\ \operatorname{Re}(1)-\mathrm{P}(3) & 2.4420(12) & \operatorname{Re}(1)-\mathrm{P}(4) & 2.4502(11) \\ \operatorname{Re}(1)-\mathrm{P}(5) & 2.4566(11) & \operatorname{Re}(1)-\mathrm{P}(6) & 2.4694(12) \\ & & & \\ \mathrm{P}(1)-\operatorname{Re}(1)-\mathrm{P}(2) & 79.85(4) & \mathrm{P}(1)-\operatorname{Re}(1)-\mathrm{P}(3) & 94.63(4) \\ \mathrm{P}(1)-\operatorname{Re}(1)-\mathrm{P}(4) & 168.26(4) & \mathrm{P}(1)-\operatorname{Re}(1)-\mathrm{P}(5) & 95.03(4) \\ \mathrm{P}(1)-\operatorname{Re}(1)-\mathrm{P}(6) & 91.03(4) & \mathrm{P}(2)-\operatorname{Re}(1)-\mathrm{P}(3) & 94.24(4) \\ \mathrm{P}(2)-\operatorname{Re}(1)-\mathrm{P}(4) & 89.69(4) & \mathrm{P}(2)-\operatorname{Re}(1)-\mathrm{P}(5) & 173.74(4) \\ \mathrm{P}(2)-\operatorname{Re}(1)-\mathrm{P}(6) & 96.47(4) & \mathrm{P}(3)-\operatorname{Re}(1)-\mathrm{P}(4) & 80.73(4) \\ \mathrm{P}(3)-\operatorname{Re}(1)-\mathrm{P}(5) & 89.74(4) & \mathrm{P}(4)-\operatorname{Re}(1)-\mathrm{P}(5) & 95.72(4) \\ \mathrm{P}(3)-\operatorname{Re}(1)-\mathrm{P}(6) & 168.62(4) & \mathrm{P}(4)-\operatorname{Re}(1)-\mathrm{P}(6) & 95.47(4) \\ \mathrm{P}(5)-\operatorname{Re}(1)-\mathrm{P}(6) & 79.92(4) & & \end{array}$


Table S16. Selected Bond Distances $(\AA)$ and Angles $\left({ }^{\circ}\right)$ for $\left[\operatorname{Re}(\mathrm{dmpm})_{3}\right]\left(\mathrm{B}\left(\mathrm{C}_{6} \mathrm{~F}_{5}\right)_{4}\right)_{2}$. $\mathrm{CH}_{2} \mathrm{Cl}_{2}(\mathbf{1 0})$.

$\begin{array}{llll}\operatorname{Re}(1)-\mathrm{P}(1) & 2.395(2) & \operatorname{Re}(1)-\mathrm{P}(2) & 2.434(2) \\ \operatorname{Re}(1)-\mathrm{P}(3) & 2.4499(18) & \operatorname{Re}(1)-\mathrm{P}(4) & 2.4301(19) \\ \operatorname{Re}(1)-\mathrm{P}(5) & 2.4091(18) & \operatorname{Re}(1)-\mathrm{P}(6) & 2.385(2) \\ & & & \\ \mathrm{P}(1)-\operatorname{Re}(1)-\mathrm{P}(2) & 67.18(8) & \mathrm{P}(1)-\operatorname{Re}(1)-\mathrm{P}(3) & 159.74(7) \\ \mathrm{P}(1)-\operatorname{Re}(1)-\mathrm{P}(4) & 100.33(7) & \mathrm{P}(1)-\operatorname{Re}(1)-\mathrm{P}(5) & 108.38(7) \\ \mathrm{P}(1)-\operatorname{Re}(1)-\mathrm{P}(6) & 100.61(8) & \mathrm{P}(2)-\operatorname{Re}(1)-\mathrm{P}(3) & 96.78(8) \\ \mathrm{P}(2)-\operatorname{Re}(1)-\mathrm{P}(4) & 98.79(8) & \mathrm{P}(2)-\operatorname{Re}(1)-\mathrm{P}(5) & 163.14(8) \\ \mathrm{P}(2)-\operatorname{Re}(1)-\mathrm{P}(6) & 95.01(9) & \mathrm{P}(3)-\operatorname{Re}(1)-\mathrm{P}(4) & 68.86(7) \\ \mathrm{P}(3)-\operatorname{Re}(1)-\mathrm{P}(5) & 90.47(6) & \mathrm{P}(3)-\operatorname{Re}(1)-\mathrm{P}(6) & 92.82(8) \\ \mathrm{P}(4)-\operatorname{Re}(1)-\mathrm{P}(6) & 158.16(8) & \mathrm{P}(4)-\operatorname{Re}(1)-\mathrm{P}(5) & 98.02(7) \\ \mathrm{P}(5)-\operatorname{Re}(1)-\mathrm{P}(6) & 69.36(8) & & \end{array}$


Table S17. Selected Bond Distances $(\AA)$ and Angles $\left({ }^{\circ}\right)$ for $\left[\mathrm{Re}\left(\mathrm{Me}_{2} \mathrm{PCH}_{2} \mathrm{PPh}_{2}\right)_{3}\right]_{2}\left(\mathrm{PF}_{6}\right)_{3}$ (11).

\begin{tabular}{lccl}
$\operatorname{Re}(1)-\mathrm{P}(1)$ & $2.3987(9)$ & \multicolumn{1}{l}{$\operatorname{Re}(1)-\mathrm{P}(1) \# 1$} & $2.3987(9)$ \\
$\operatorname{Re}(1)-\mathrm{P}(1) \# 2$ & $2.3987(9)$ & $\operatorname{Re}(1)-\mathrm{P}(2)$ & $2.4646(8)$ \\
$\operatorname{Re}(1)-\mathrm{P}(2) \# 1$ & $2.4646(8)$ & $\mathrm{Re}(1)-\mathrm{P}(2) \# 2$ & $2.4646(8)$ \\
& & & \\
& & & \\
$\mathrm{P}(1) \# 1-\operatorname{Re}(1)-\mathrm{P}(1)$ & $93.20(3)$ & $\mathrm{P}(1) \# 1-\operatorname{Re}(1)-\mathrm{P}(1) \# 2$ & $93.20(3)$ \\
$\mathrm{P}(1)-\operatorname{Re}(1)-\mathrm{P}(1) \# 2$ & $93.20(3)$ & $\mathrm{P}(1) \# 1-\operatorname{Re}(1)-\mathrm{P}(2)$ & $93.21(3)$ \\
$\mathrm{P}(1)-\operatorname{Re}(1)-\mathrm{P}(2)$ & $68.66(3)$ & $\mathrm{P}(1) \# 2-\operatorname{Re}(1)-\mathrm{P}(2)$ & $161.06(3)$ \\
$\mathrm{P}(1) \# 1-\operatorname{Re}(1)-\mathrm{P}(2) \# 1$ & $68.66(3)$ & $\mathrm{P}(1)-\operatorname{Re}(1)-\mathrm{P}(2) \# 1$ & $161.06(3)$ \\
$\mathrm{P}(1) \# 2-\operatorname{Re}(1)-\mathrm{P}(2) \# 1$ & $93.21(3)$ & $\mathrm{P}(2)-\operatorname{Re}(1)-\mathrm{P}(2) \# 1$ & $105.72(2)$ \\
$\mathrm{P}(1) \# 1-\operatorname{Re}(1)-\mathrm{P}(2) \# 2$ & $161.06(3)$ & $\mathrm{P}(1)-\operatorname{Re}(1)-\mathrm{P}(2) \# 2$ & $93.21(3)$ \\
$\mathrm{P}(1) \# 2-\operatorname{Re}(1)-\mathrm{P}(2) \# 2$ & $68.66(3)$ & $\mathrm{P}(2)-\operatorname{Re}(1)-\mathrm{P}(2) \# 2$ & $105.72(2)$ \\
$\mathrm{P}(2) \# 1-\operatorname{Re}(1)-\mathrm{P}(2) \# 2$ & $105.72(2)$ & &
\end{tabular}


Table S18. Selected Bond Distances $(\AA)$ and Angles $\left({ }^{\circ}\right)$ for $\left[\operatorname{Re}(\mathrm{dmpb})_{3}\right]\left(\mathrm{SbF}_{6}\right)_{2} \cdot \mathrm{PhCl}(\mathbf{1 4})$.

$\begin{array}{llll}\operatorname{Re}(1)-\mathrm{P}(1) & 2.4253(11) & \mathrm{Re}(2)-\mathrm{P}(7) & 2.4233(13) \\ \operatorname{Re}(1)-\mathrm{P}(2) & 2.4335(12) & \operatorname{Re}(2)-\mathrm{P}(8) & 2.4257(13) \\ \operatorname{Re}(1)-\mathrm{P}(3) & 2.4276(13) & \operatorname{Re}(2)-\mathrm{P}(9) & 2.4215(13) \\ \operatorname{Re}(1)-\mathrm{P}(4) & 2.4196(11) & \operatorname{Re}(2)-\mathrm{P}(10) & 2.4263(13) \\ \operatorname{Re}(1)-\mathrm{P}(5) & 2.4192(13) & \operatorname{Re}(2)-\mathrm{P}(11) & 2.4198(13) \\ \operatorname{Re}(1)-\mathrm{P}(6) & 2.4222(13) & \mathrm{Re}(2)-\mathrm{P}(12) & 2.4346(13) \\ & & & \\ \mathrm{P}(1)-\operatorname{Re}(1)-\mathrm{P}(2) & 81.64(4) & \mathrm{P}(7)-\operatorname{Re}(2)-\mathrm{P}(8) & 81.90(5) \\ \mathrm{P}(1)-\operatorname{Re}(1)-\mathrm{P}(3) & 92.26(4) & \mathrm{P}(7)-\operatorname{Re}(2)-\mathrm{P}(9) & 90.78(5) \\ \mathrm{P}(1)-\operatorname{Re}(1)-\mathrm{P}(4) & 172.05(4) & \mathrm{P}(7)-\operatorname{Re}(2)-\mathrm{P}(10) & 170.34(5) \\ \mathrm{P}(1)-\operatorname{Re}(1)-\mathrm{P}(5) & 94.37(4) & \mathrm{P}(7)-\operatorname{Re}(2)-\mathrm{P}(11) & 94.10(5) \\ \mathrm{P}(1)-\operatorname{Re}(1)-\mathrm{P}(6) & 93.87(4) & \mathrm{P}(7)-\operatorname{Re}(2)-\mathrm{P}(12) & 98.26(5) \\ \mathrm{P}(2)-\operatorname{Re}(1)-\mathrm{P}(3) & 96.72(4) & \mathrm{P}(8)-\operatorname{Re}(2)-\mathrm{P}(9) & 95.82(5) \\ \mathrm{P}(2)-\operatorname{Re}(1)-\mathrm{P}(4) & 93.38(4) & \mathrm{P}(8)-\operatorname{Re}(2)-\mathrm{P}(10) & 92.17(4) \\ \mathrm{P}(2)-\operatorname{Re}(1)-\mathrm{P}(5) & 171.39(5) & \mathrm{P}(8)-\operatorname{Re}(2)-\mathrm{P}(11) & 173.36(4) \\ \mathrm{P}(2)-\operatorname{Re}(1)-\mathrm{P}(6) & 90.81(4) & \mathrm{P}(8)-\operatorname{Re}(2)-\mathrm{P}(12) & 93.43(4) \\ \mathrm{P}(3)-\operatorname{Re}(1)-\mathrm{P}(4) & 82.12(4) & \mathrm{P}(9)-\operatorname{Re}(2)-\mathrm{P}(10) & 82.20(4) \\ \mathrm{P}(3)-\operatorname{Re}(1)-\mathrm{P}(5) & 91.03(5) & \mathrm{P}(9)-\operatorname{Re}(2)-\mathrm{P}(11) & 89.51(4) \\ \mathrm{P}(3)-\operatorname{Re}(1)-\mathrm{P}(6) & 170.90(4) & \mathrm{P}(9)-\operatorname{Re}(2)-\mathrm{P}(12) & 167.90(5) \\ \mathrm{P}(4)-\operatorname{Re}(1)-\mathrm{P}(5) & 91.36(4) & \mathrm{P}(10)-\operatorname{Re}(2)-\mathrm{P}(11) & 92.48(4) \\ \mathrm{P}(4)-\operatorname{Re}(1)-\mathrm{P}(6) & 92.39(4) & \mathrm{P}(10)-\operatorname{Re}(2)-\mathrm{P}(12) & 89.67(4) \\ \mathrm{P}(5)-\operatorname{Re}(1)-\mathrm{P}(6) & 81.81(5) & \mathrm{P}(11)-\operatorname{Re}(2)-\mathrm{P}(12) & 81.86(4)\end{array}$


Table S19. Cartesian coordinates (in Å) of optimized geometry for (dmpe) Re $_{3}^{+}$(DFT M06-L functional, cc-pVDz atom basis sets)

\begin{tabular}{|c|c|c|c|}
\hline & 61 & 73 & -0 . \\
\hline & 6 & (1) & (1) \\
\hline & 1.849447950307 & 0.006394210550 & 1.520332850721 \\
\hline$P$ & -1.379765416454 & 1.397374953496 & 1.374421349601 \\
\hline & 00434373 & 2.126240173633 & -0.9779074 \\
\hline D & -1.843566083652 & -0.454973511353 & -1.506019 \\
\hline P & 1.646097227788 & -1.047791694158 & 00430 \\
\hline & 700960 & 7210 & 575 \\
\hline $\mathrm{H}$ & -1.768523575394 & -3.405478872712 & -0.75868 \\
\hline $\mathrm{H}$ & -2.860842822723 & -3.394796886188 & 0.628216678556 \\
\hline$C$ & 03892 & -1.646508953096 & -0.67 \\
\hline $\mathrm{H}$ & -3.728774320080 & -2.040189852520 & -1.4 \\
\hline $\mathrm{H}$ & -3.593129017424 & -1.072736343441 & 0.067295872710 \\
\hline $\mathrm{C}$ & 3. & -0 . & 937 \\
\hline $\mathrm{H}$ & 4.229 & -0.62 & 1.2 \\
\hline $\mathrm{H}$ & 3.142068492490 & -1.989593975954 & 1.018493761275 \\
\hline $\mathrm{C}$ & 3.336877874469 & -0.71 & -0.71 \\
\hline $\mathrm{H}$ & 4.081703684458 & 127 & 35 \\
\hline $\mathrm{H}$ & 3.611417764219 & 0.318202988082 & -0.966573665059 \\
\hline$C$ & 0.076408689815 & 3.480780 & 0.202902 \\
\hline & 0 & 4.4 & -0 \\
\hline $\mathrm{H}$ & 0.8413832635 & 3.527 & 0.994070500288 \\
\hline $\mathrm{C}$ & -1.287143031109 & 3.167622436178 & 0.783360139022 \\
\hline$x$ & -2.067055866016 & 3.2862684337 & 5012852215 \\
\hline$\Pi$ & -1.557602345285 & 3.849265946774 & 43986 \\
\hline $\mathrm{C}$ & 1.783748729082 & -0.795066743231 & 3.181279857134 \\
\hline 11 & 2 . & -0.8 & 2 \\
\hline H & 1.111751649646 & -0.240303333009 & 3.848770673697 \\
\hline
\end{tabular}




\begin{tabular}{llll} 
H & 1.426507332908 & -1.830780421892 & 3.117973128786 \\
$\mathrm{C}$ & 2.687148704443 & 1.566519358783 & 2.054108062519 \\
$\mathrm{H}$ & 1.999641799917 & 2.195057570387 & 2.634882628047 \\
$\mathrm{H}$ & 3.560847476441 & 1.348303251112 & 2.684218072620 \\
$\mathrm{H}$ & 3.021967320556 & 2.151741156595 & 1.190808155523 \\
$\mathrm{C}$ & 1.871563620864 & -0.539002255235 & -3.188565888344 \\
$\mathrm{H}$ & 2.902941680829 & -0.729615487618 & -3.519295082821 \\
$\mathrm{H}$ & 1.202141189958 & -1.123543651191 & -3.832540113644 \\
$\mathrm{H}$ & 1.641519824671 & 0.522698785094 & -3.340064428261 \\
$\mathrm{C}$ & 1.796639844326 & -2.874239077255 & -1.673600086543 \\
$\mathrm{H}$ & 0.838280145629 & -3.317650110138 & -1.976479892996 \\
$\mathrm{H}$ & 2.542429748806 & -3.099615811727 & -2.449385174853 \\
$\mathrm{H}$ & 2.117227485810 & -3.355917861537 & -0.741676609796 \\
$\mathrm{C}$ & 0.197099817093 & -3.554954127343 & 1.226144270389 \\
$\mathrm{H}$ & 1.204446191683 & -3.311800026979 & 1.588258631639 \\
$\mathrm{H}$ & -0.270414389594 & -4.248071851973 & 1.939145909928 \\
$\mathrm{H}$ & 0.303153435761 & -4.077753677553 & 0.268827374353 \\
$\mathrm{C}$ & -1.602038754852 & -1.969247075632 & 2.680388395579 \\
$\mathrm{H}$ & -2.016717807867 & -2.947871053588 & 2.960361856986 \\
$\mathrm{H}$ & -0.860271967648 & -1.680925732526 & 3.436188887110 \\
$\mathrm{H}$ & -2.413875970925 & -1.231610916512 & 2.703018677192 \\
$\mathrm{C}$ & -3.068146462796 & 0.773361355600 & -2.156771795668 \\
$\mathrm{H}$ & -2.714785063452 & 1.190554322003 & -3.108096244130 \\
$\mathrm{H}$ & -4.032451686771 & 0.280730076912 & -2.348315041533 \\
$\mathrm{H}$ & -3.236919058803 & 1.608325893098 & -1.465238167953 \\
$\mathrm{C}$ & -1.547710155088 & -1.348047094441 & -3.093685714963 \\
$\mathrm{H}$ & -2.494019893744 & -1.654874932904 & -3.562238281161 \\
$\mathrm{H}$ & -1.022056238774 & -0.685740724622 & -3.793937888827 \\
& -0.927144783992 & -2.240259328251 & -2.934698141860 \\
-3.216035905447 & 1.261939743792 & 1.536032454699 \\
\hline & -3.614376916625 & 2.145767542710 & 2.055216173432
\end{tabular}




$\begin{array}{lrll}\mathrm{H} & -3.699086774245 & 1.195026724739 & 0.553850115886 \\ \mathrm{H} & -3.505168587661 & 0.377101731220 & 2.117322950849 \\ \mathrm{C} & -0.990678424992 & 1.614329150135 & 3.167280480796 \\ \mathrm{H} & 0.045288149400 & 1.952471313655 & 3.300646048407 \\ \mathrm{H} & -1.658133845025 & 2.353548840274 & 3.632775653057 \\ \mathrm{H} & -1.108834230013 & 0.661622190324 & 3.699769345695 \\ \mathrm{C} & 2.257198935220 & 2.687735221946 & -1.456462124097 \\ \mathrm{H} & 2.613461532634 & 2.145485757525 & -2.341894629119 \\ \mathrm{H} & 2.237858006714 & 3.758635412097 & -1.704182139591 \\ \mathrm{H} & 2.989991240460 & 2.538362204390 & -0.656200130546 \\ \mathrm{C} & -0.314430547428 & 2.714389369635 & -2.494165136023 \\ \mathrm{H} & -0.182346150219 & 2.006772355141 & -3.324255784115 \\ \mathrm{H} & -1.388864919689 & 2.805927518834 & -2.296617089606 \\ \mathrm{H} & 0.063160164328 & 3.696648774800 & -2.812050820429\end{array}$


Table S20. Cartesian coordinates (in $\AA$ ) of optimized geometry for (dmpe) ${ }_{3} \mathrm{Re}^{2+}$ (DFT M06$\mathrm{L}$ functional, cc-pVDz atom basis sets)

$\begin{array}{cccc}\text { Re } & 0.007456199686 & -0.011470722596 & -0.003740438429 \\ \text { P } & -0.815440937397 & -2.082032190791 & 1.085981766429 \\ \text { P } & 1.979647433669 & 0.118858695780 & 1.474611359175 \\ \text { P } & -1.412744740617 & 1.521685510098 & 1.316912445517 \\ \text { P } & 0.575229622406 & 2.141816667404 & -1.076893924813 \\ \text { P } & -1.979888711622 & -0.512902176721 & -1.412126432543 \\ \text { P } & 1.659638466765 & -1.188691896073 & -1.413639958157 \\ \text { C } & -2.236894570759 & -2.787024837668 & 0.134138895763 \\ \text { H } & -1.813102564833 & -3.431986193620 & -0.651657611827 \\ \text { H } & -2.833509573856 & -3.442477115127 & 0.785530237657 \\ \text { C } & -3.069281812147 & -1.678918977553 & -0.475733591499 \\ \text { H } & -3.845025762479 & -2.072955927332 & -1.149084911024 \\ \text { H } & -3.588520311841 & -1.098462010707 & 0.302405084277 \\ \text { C } & 3.363903031455 & -0.868785378718 & 0.731835629531 \\ \text { H } & 4.322949726248 & -0.540598239455 & 1.158683939250 \\ \text { H } & 3.224678056899 & -1.914295537436 & 1.050876127414 \\ \text { C } & 3.347918147407 & -0.767124532399 & -0.781227802978 \\ \text { H } & 4.091602068929 & -1.433393708410 & -1.243469620631 \\ \text { H } & 3.589391239018 & 0.253661513981 & -1.113217845151 \\ \mathrm{C} & 0.074359291983 & 3.520397881794 & 0.048852310442 \\ \text { H } & 0.097181717282 & 4.472489332416 & -0.502615174894 \\ \text { H } & 0.833052545895 & 3.597868140521 & 0.842587274472 \\ \text { C } & -1.299367003160 & 3.239458581474 & 0.624487966711 \\ \text { H } & -2.074054013872 & 3.328327186014 & -0.153807843105 \\ \text { H } & -1.571869291087 & 3.962755070375 & 1.407366277860 \\ \text { C } & 1.866064331947 & -0.618886812780 & 3.152005954433 \\ \text { H } & 2.859653173836 & -0.613084471968 & 3.621397084403 \\ \text { H } & 1.188703444527 & -0.041442820008 & 3.792727641793\end{array}$




\begin{tabular}{llll} 
H & 1.516077710048 & -1.657067070563 & 3.112158722605 \\
C & 2.812724425262 & 1.698926887857 & 1.914498455464 \\
H & 2.129607795686 & 2.369296218088 & 2.450754869122 \\
H & 3.669137402477 & 1.496297609643 & 2.571867413932 \\
H & 3.184185765028 & 2.228206415535 & 1.030975586995 \\
C & 1.761282735510 & -0.726997854816 & -3.190195849320 \\
H & 2.773997302127 & -0.930220392655 & -3.565785275769 \\
H & 1.062717180242 & -1.335598940575 & -3.776636197695 \\
H & 1.529874323175 & 0.330130977244 & -3.366820648848 \\
$\mathrm{C}$ & 1.792166627970 & -3.014231112525 & -1.564861935442 \\
$\mathrm{H}$ & 0.830005310672 & -3.469111415867 & -1.834321041714 \\
$\mathrm{H}$ & 2.521293185489 & -3.265753829240 & -2.347472919183 \\
$\mathrm{H}$ & 2.138032419812 & -3.458674765739 & -0.624487646313 \\
$\mathrm{C}$ & 0.197278670633 & -3.590708710426 & 1.365783098444 \\
$\mathrm{H}$ & 1.187623201533 & -3.357771109912 & 1.777215209481 \\
$\mathrm{H}$ & -0.316643687339 & -4.258823011415 & 2.070026176020 \\
$\mathrm{H}$ & 0.333973063572 & -4.138185583433 & 0.427041450025 \\
$\mathrm{C}$ & -1.542152270060 & -1.853229407481 & 2.759738533239 \\
$\mathrm{H}$ & -1.937835478617 & -2.807197407218 & 3.134021368778 \\
$\mathrm{H}$ & -0.787135684346 & -1.495768583746 & 3.470566865021 \\
$\mathrm{H}$ & -2.364402540353 & -1.127417699343 & 2.735229042021 \\
$\mathrm{C}$ & -3.195632475805 & 0.710358894882 & -2.061988558616 \\
$\mathrm{H}$ & -2.879850587753 & 1.058987831855 & -3.053013768835 \\
$\mathrm{H}$ & -4.177082133255 & 0.229908124789 & -2.179664475477 \\
$\mathrm{H}$ & -3.317159802408 & 1.588266125348 & -1.417119256106 \\
$\mathrm{C}$ & -1.662859678578 & -1.455742792925 & -2.954741078040 \\
$\mathrm{H}$ & -2.607735384541 & -1.786215170099 & -3.408052879026 \\
& -1.143244657605 & -0.820424386849 & -3.683003895378 \\
\hline & -1.039118622873 & -2.335817527676 & -2.753315154589 \\
$\mathrm{H}$ & -3.613230567183 & 2.285291043657 & 2.019162209587
\end{tabular}




$\begin{array}{lrll}\mathrm{H} & -3.759786903341 & 1.234690203897 & 0.594559377874 \\ \mathrm{H} & -3.481932565126 & 0.530059315932 & 2.203784725213 \\ \mathrm{C} & -0.915402161588 & 1.772263395074 & 3.068812573171 \\ \mathrm{H} & 0.132227616717 & 2.090146498971 & 3.139268107066 \\ \mathrm{H} & -1.543905846316 & 2.542676366392 & 3.536299393667 \\ \mathrm{H} & -1.036331438993 & 0.843884293927 & 3.640818477316 \\ \mathrm{C} & 2.255616643318 & 2.652966603350 & -1.617500498425 \\ \mathrm{H} & 2.577933599685 & 2.064628249238 & -2.485668570543 \\ \mathrm{H} & 2.234555243893 & 3.708886114021 & -1.920595535615 \\ \mathrm{H} & 3.009020799598 & 2.541092339042 & -0.831452233732 \\ \mathrm{C} & -0.363219361999 & 2.555871781055 & -2.601562629353 \\ \mathrm{H} & -0.251498994937 & 1.775060260615 & -3.365320601000 \\ \mathrm{H} & -1.429582774816 & 2.657842077651 & -2.371200821408 \\ \mathrm{H} & -0.008405299462 & 3.504658389036 & -3.026740930390\end{array}$


B. Absorption Spectra of Complexes

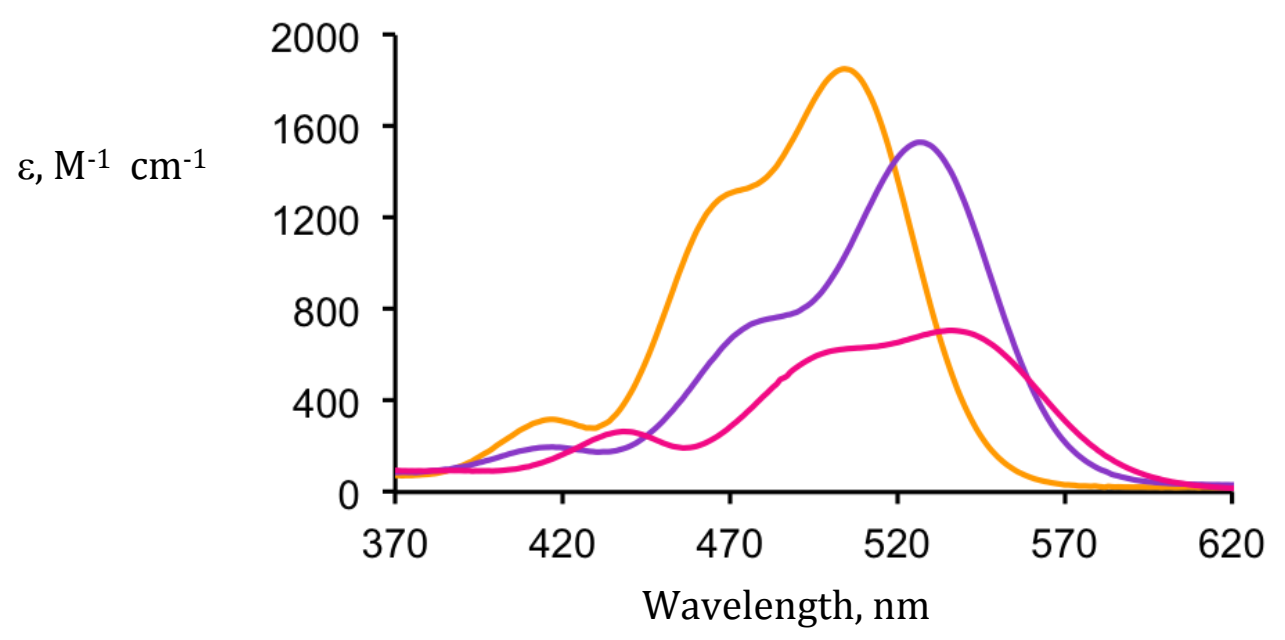

Figure S1. UV-Vis spectra of $(\mathrm{PP})_{3} \mathrm{Re}^{2+}$ in $\mathrm{CH}_{3} \mathrm{CN}(\mathrm{PP}=\operatorname{dmpm}(-)$, dmpe (-), dmpp (-).

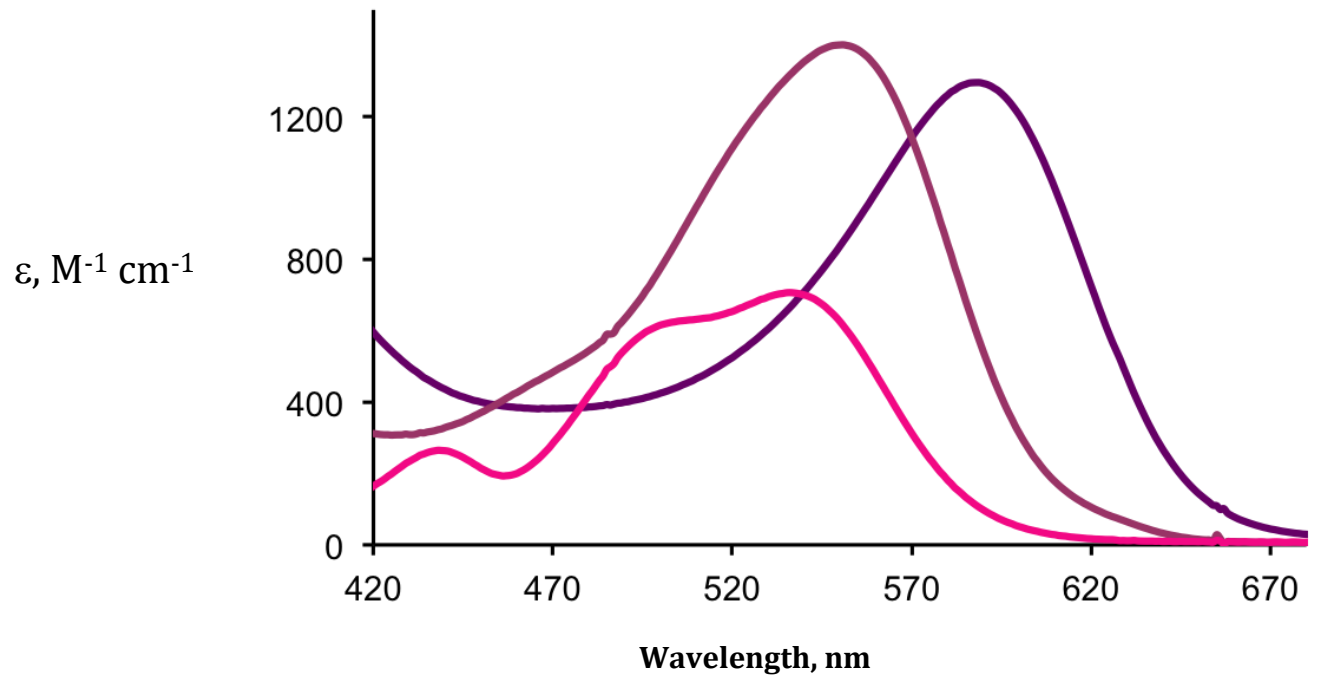

Figure S2. UV-Vis spectra of $(\mathrm{PP})_{3} \mathrm{Re}^{2+}$ in $\mathrm{CH}_{3} \mathrm{CN}\left(\mathrm{PP}=\operatorname{dmpm}(-), \mathrm{Me}_{2} \mathrm{PCH}_{2} \mathrm{PPh}_{2}(-)\right.$, dppm $(-)$. 


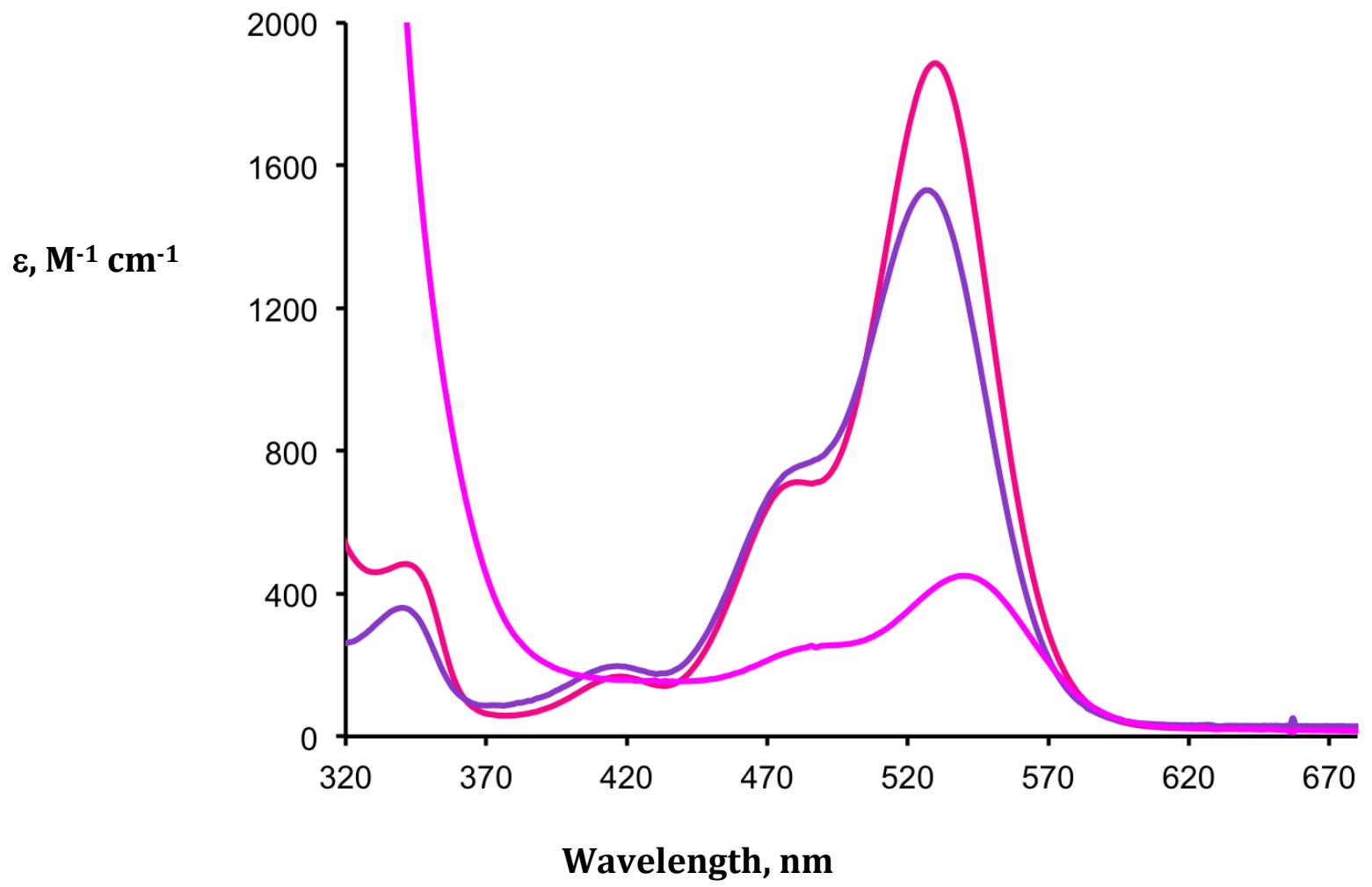

Figure S3. UV-Vis spectra of $(\mathrm{PP})_{3} \mathrm{Re}^{2+}$ in $\mathrm{CH}_{3} \mathrm{CN}(\mathrm{PP}=\operatorname{dmpe}(-)$, depe $(-), \operatorname{dmpb}(-)$. 
C. Room temperature Luminescence spectra

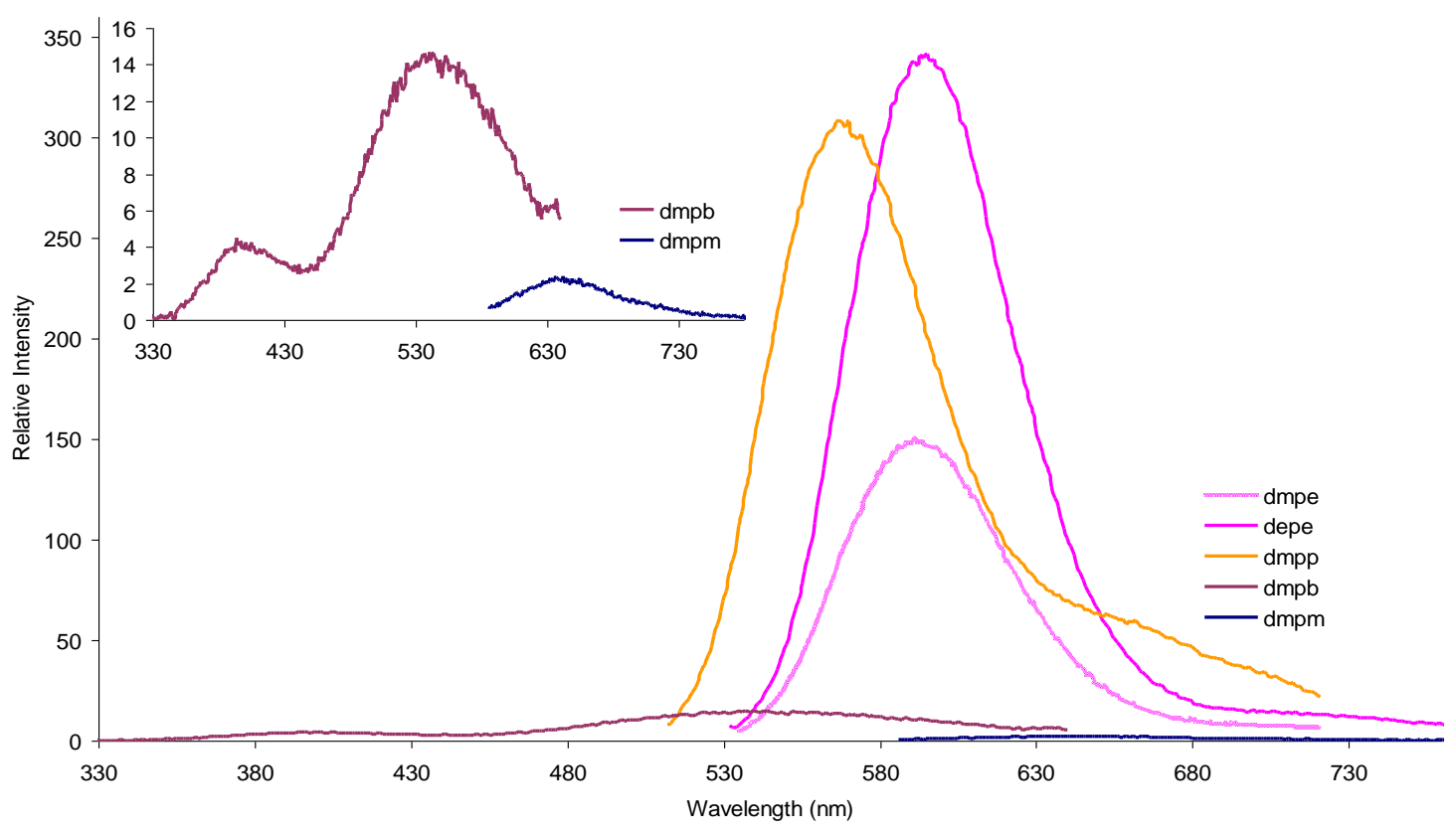

Figure S4. Uncorrected emission spectra for a deoxygenated $0.23 \mathrm{mM}$ solution of $\left[(\mathrm{PP})_{3} \mathrm{Re}\right]^{2+}$, for phosphines containing methyl or ethyl phosphine substituents, in $\mathrm{CH}_{3} \mathrm{CN}$ at room temperature. The inset shows an expanded view for the more weakly emissive complexes were $\mathrm{PP}=\mathrm{dmpm}$ and $\mathrm{dmpb}$. 
D. Cyclic Voltammetric Data for the $\mathrm{Re}^{2+} /{ }^{+}$process of the complexes.

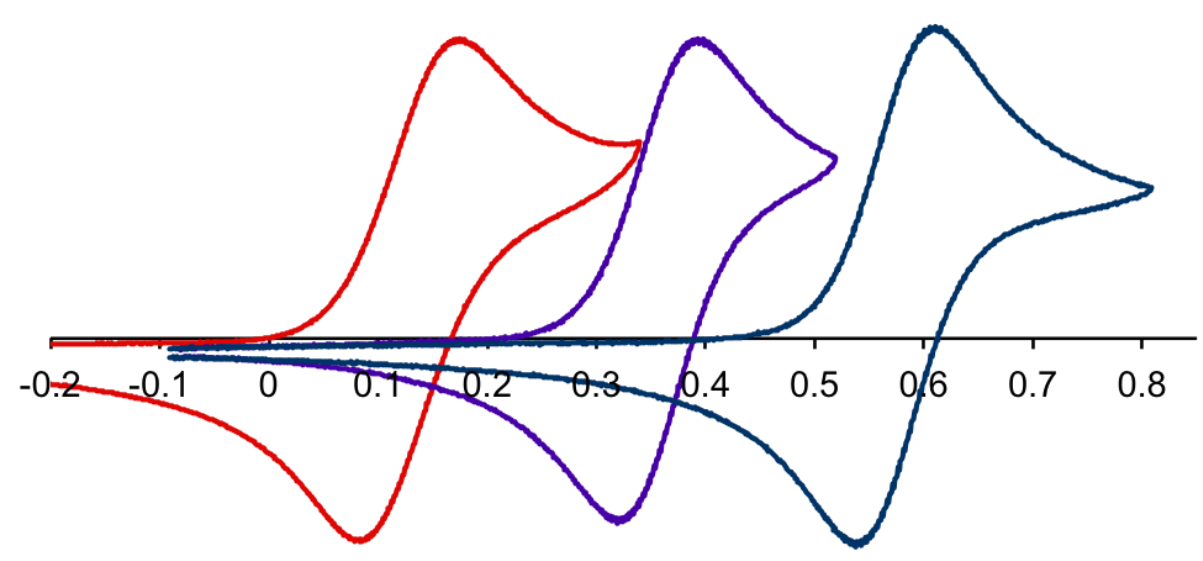

Figure S5. Cyclic Voltammetry for (PP) ${ }_{3} \mathrm{Re}^{+}$complexes ( $\mathrm{PP}=\operatorname{dmpm}(-), \mathrm{Me}_{2} \mathrm{PCH}_{2} \mathrm{PPh}_{2}(-)$, dppm (-) in $1 \mathrm{mM} \mathrm{CH}_{3} \mathrm{CN}$, scan rate $100 \mathrm{mV}, 0.1 \mathrm{M} \mathrm{TBAPF}_{6}$, versus $\mathrm{Ag} / \mathrm{Ag}^{+}(\mathrm{MeCN})$.

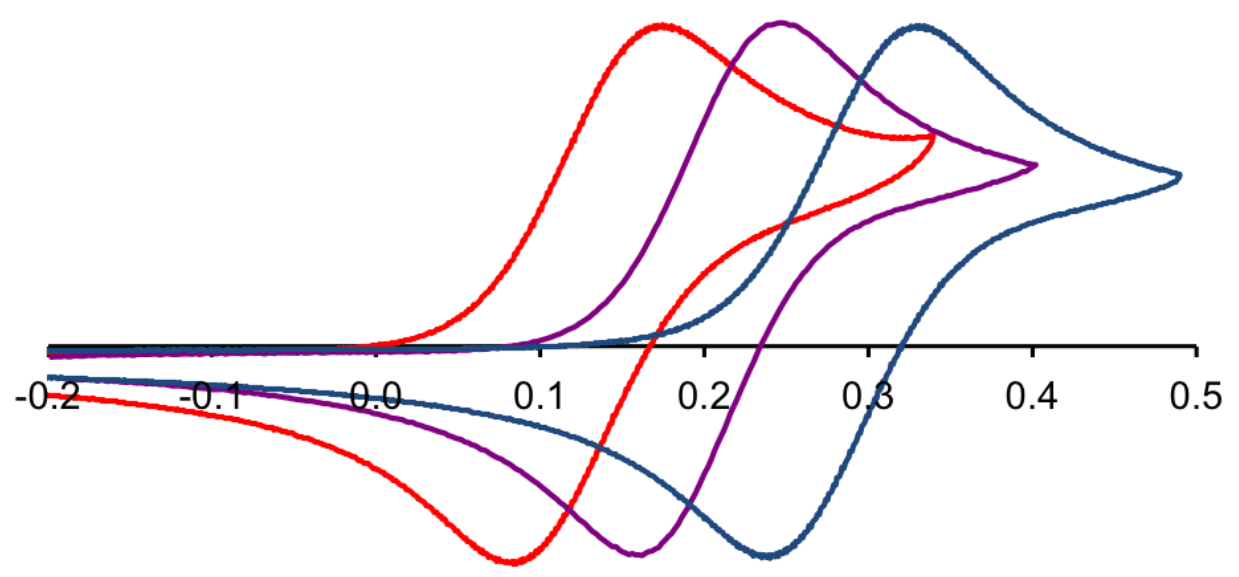

Figure S6. Cyclic Voltammetry for (PP) ${ }_{3} \mathrm{Re}^{+}$complexes (PP $=\operatorname{dmpm}(-)$,dmpp (-), dmpe (-) in $1 \mathrm{mM} \mathrm{CH}_{3} \mathrm{CN}$, scan rate $100 \mathrm{mV}, 0.1 \mathrm{M} \mathrm{TBAPF}_{6}$, versus $\mathrm{Ag} / \mathrm{Ag}^{+}(\mathrm{MeCN})$. 


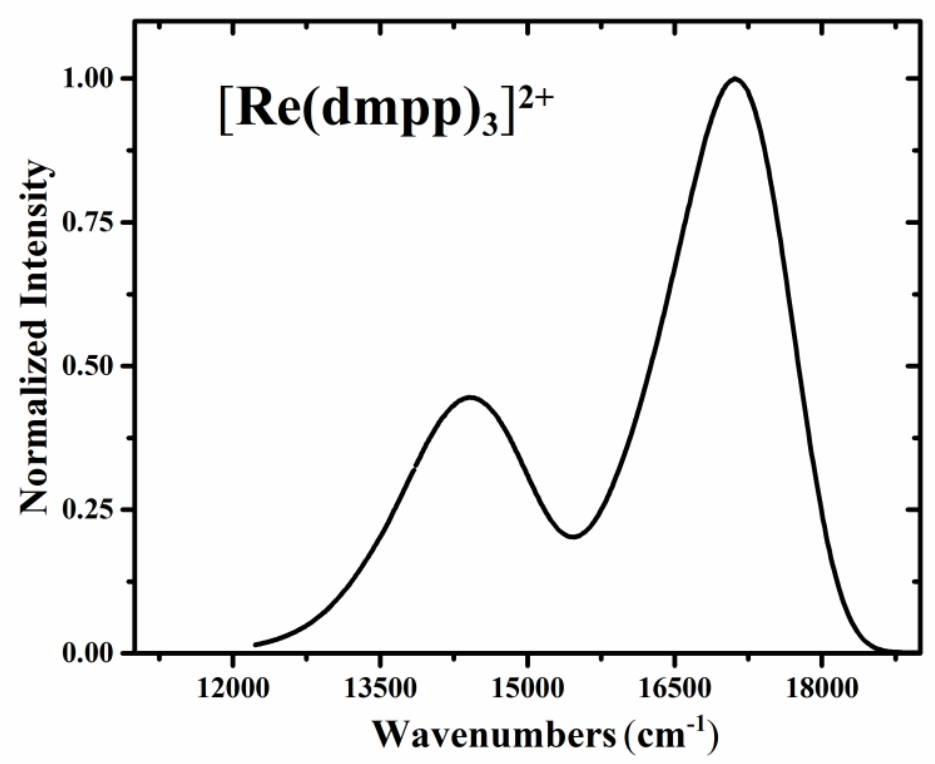

Figure S7. $77 \mathrm{~K}$ luminescence spectrum of $\left[\operatorname{Re}(\mathrm{dmpp})_{3}\right]^{2+}$ in a mixed propionitrile/ butyronitrile matrix.

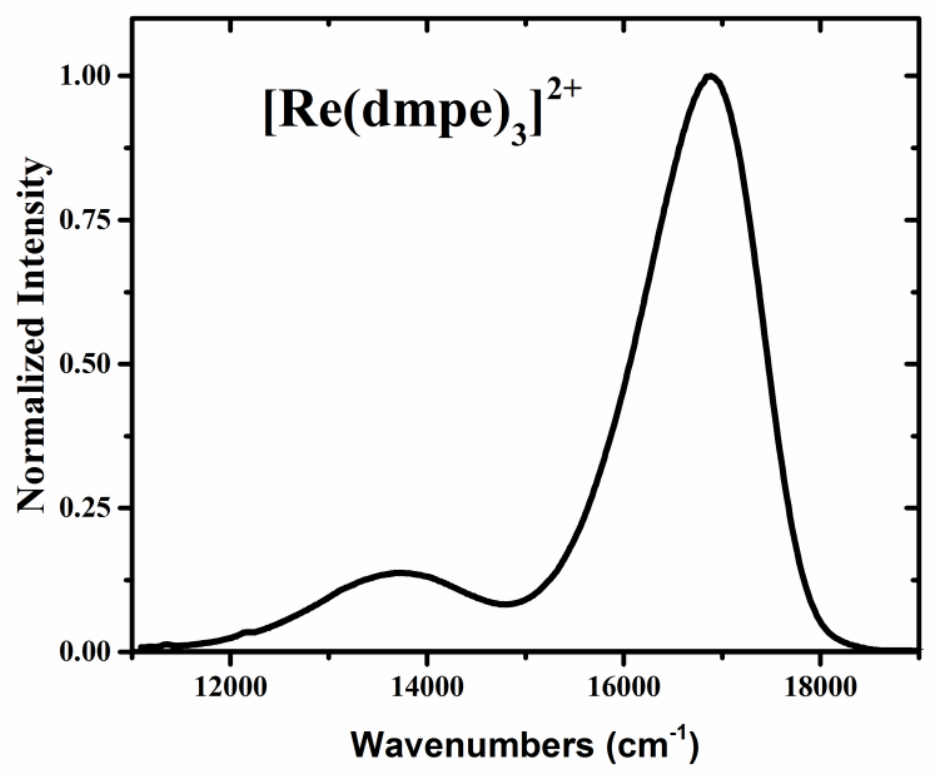

Figure S8. $77 \mathrm{~K}$ luminescence spectrum of $\left[\operatorname{Re}(\mathrm{dmpe})_{3}\right]^{2+}$ in a mixed propionitrile/ butyronitrile matrix. 


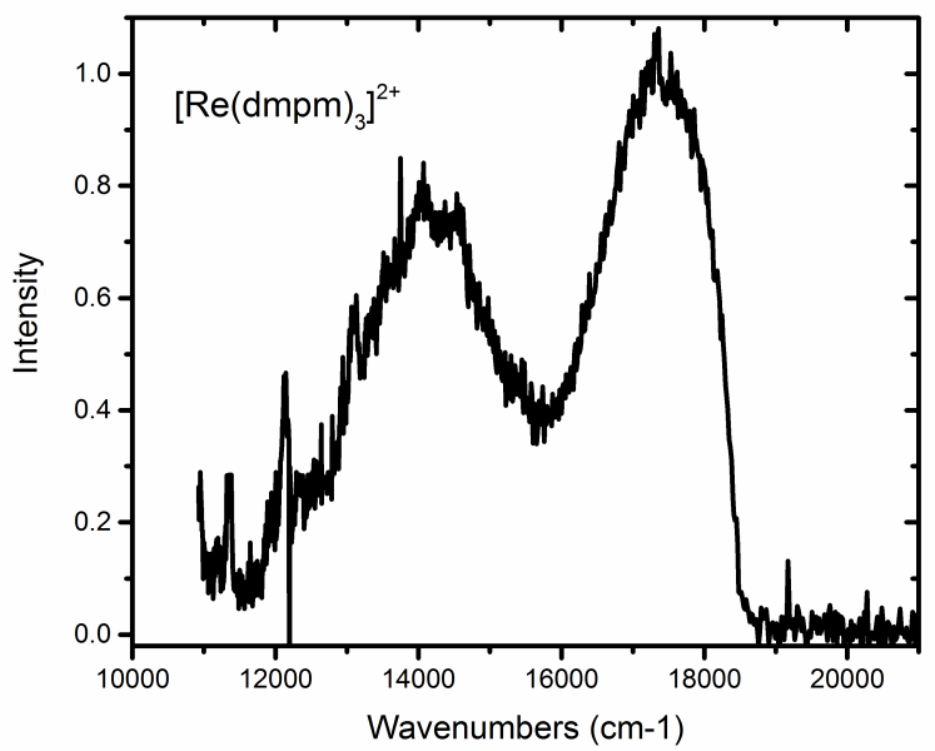

Figure S9. $77 \mathrm{~K}$ luminescence spectrum of $\left[\operatorname{Re}(\mathrm{dmpm})_{3}\right]^{2+}$ in a mixed propionitrile/ butyronitrile matrix.

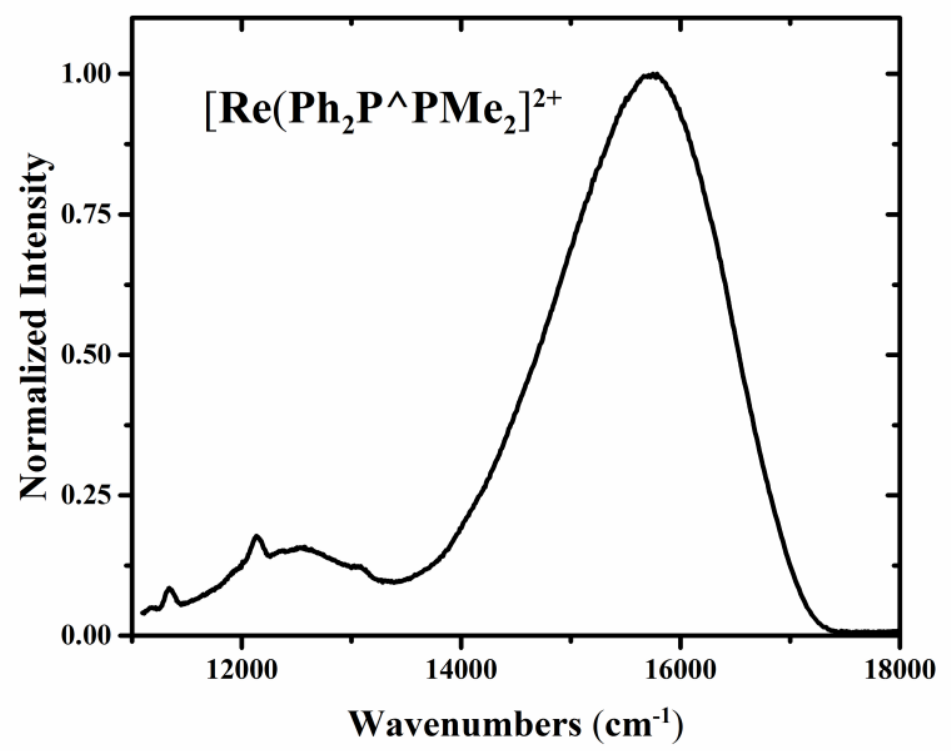

Figure S10. $77 \mathrm{~K}$ luminescence spectrum of $\left[\mathrm{Re}\left(\mathrm{Ph}_{2} \mathrm{PCH}_{2} \mathrm{PMe}_{2}\right)_{3}\right]^{2+}$ in a mixed propionitrile/ butyronitrile matrix. 


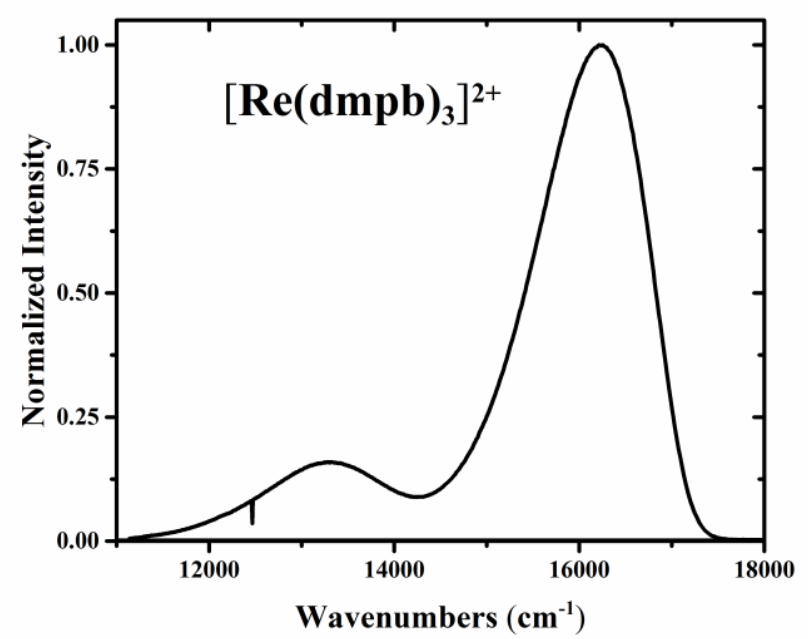

Figure S11. $77 \mathrm{~K}$ luminescence spectrum of $\left[\operatorname{Re}(\mathrm{dmpb})_{3}\right]^{2+}$ in a mixed propionitrile/ butyronitrile matrix. 


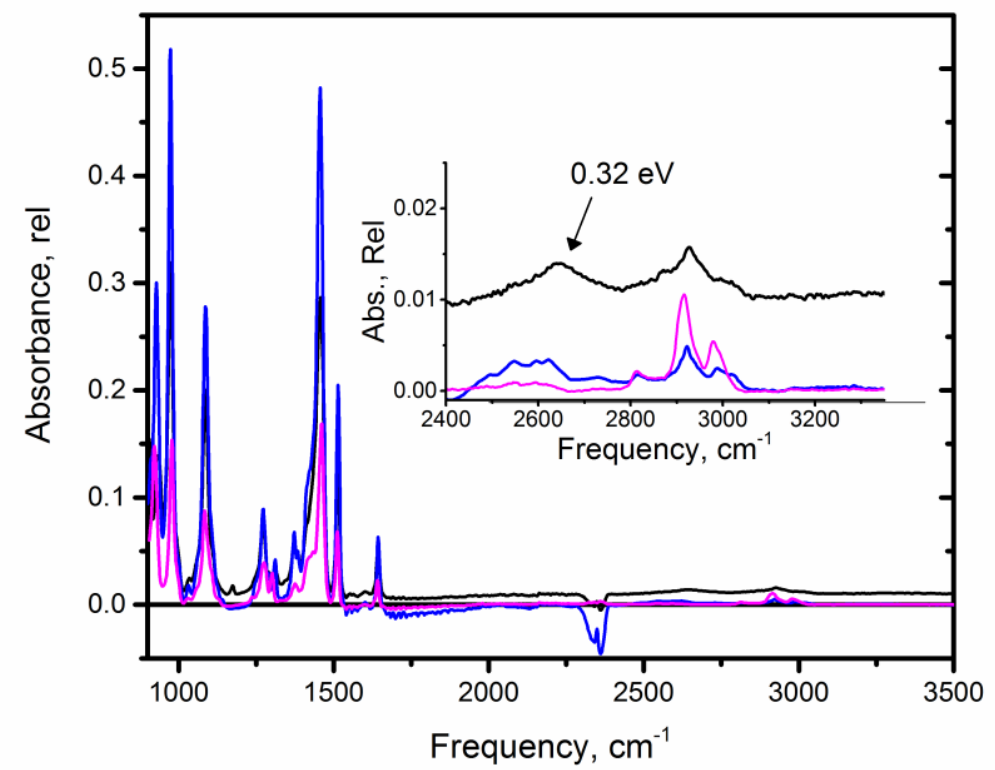

F. Figure S12. Infrared spectra of $\left[\operatorname{Re}(d m p e)_{3}\right]^{+}$(magenta), $\left[\operatorname{Re}(d m p e)_{3}\right]^{2+}$ (blue) and $\left[\operatorname{Re}(\mathrm{dmpp})_{3}\right]^{2+}($ black $)$.

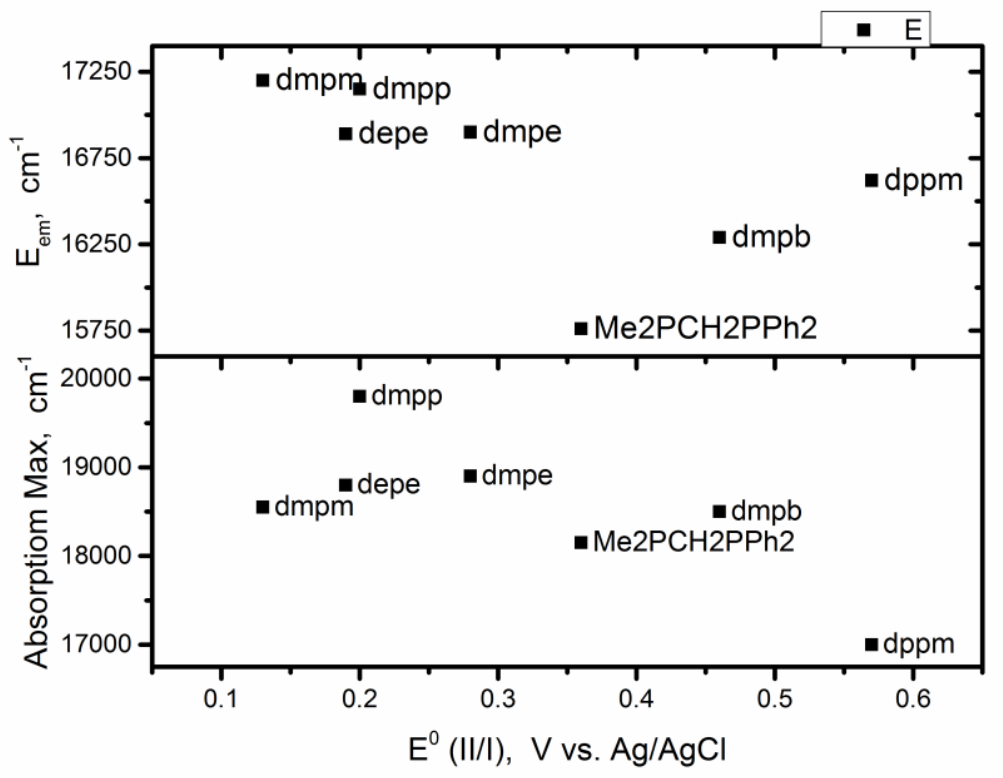

G. Figure S13. Plot of the luminescence maxima and absorption maxima of the complexes as a function of $\mathrm{E}^{0}\left(\mathrm{Re}^{2+/+}\right)$. 
H. Table S19. Luminescence quenching rate constants for $\left[\operatorname{Re}\left(\mathrm{depe}_{3}\right]^{2+}\right.$ by a series of amines, alkoxybenzenes and simple aromatics.

\begin{tabular}{lcc}
\hline Quencher & $\mathbf{E}_{\mathbf{o x}}(\mathbf{V})$ & $\mathbf{k}_{\mathbf{q}}\left(\mathbf{M}^{-1} \mathbf{s}^{-\mathbf{1}}\right)$ \\
& $\mathbf{v s} \mathbf{S C E}$ & \\
\hline 10-methylphenothiazine & 0.54 & $3.3 \times 10^{10}$ \\
Dimethylaniline & 0.71 & $2.9 \times 10^{10}$ \\
Methylphenothiazine & 0.76 & $2.6 \times 10^{10}$ \\
1,2,4-trimethoxybenzene & 1.12 & $1.6 \times 10^{10}$ \\
1,4-dimethoxybenzene & 1.34 & $1.4 \times 10^{10}$ \\
1,2-dimethoxybenzene & 1.45 & $2.3 \times 10^{10}$ \\
2-methoxynaphthalene & 1.52 & $1.8 \times 10^{10}$ \\
Anisole & 1.76 & $1.1 \times 10^{10}$ \\
M-Xylene & 2.10 & $1.1 \times 10^{10}$ \\
Toluene & 2.30 & $9.0 \times 10^{7}$ \\
\hline
\end{tabular}

